\title{
Phenotypic trait changes in laboratory - reared colonies of the maize herbivore, Diabrotica virgifera virgifera
}

\author{
H. Li ${ }^{1,2}$, T. Guillemaud ${ }^{3}$, B. W. French ${ }^{4}$, U. Kuhlmann ${ }^{2}$ \\ and S. Toepfer ${ }^{2,5 *}$
}

${ }^{1}$ Chinese Ministry of Agriculture - CABI Joint Laboratory for Biosafety, Yuanmingyuan Western Road 2, CN - 100193 Beijing, People's Republic of China: ${ }^{2} \mathrm{CABI}$, Rue des Grillons 1, CH - 2800 Delémont, Switzerland:

${ }^{3}$ INRA UMR, Route des Chappes 400, F - 06903 Sophia Antipolis, France:

${ }^{4}$ USDA ARS, North Central Agricultural Research Laboratory, Medary Avenue 2923, US - 57006 Brookings, South Dakota, USA: ${ }^{5}$ CABI, c/o Plant Protection Directorate, Rarosi ut 110, H - 6800 Hodmezovasarhely, Hungary

\begin{abstract}
The North American and European maize pest Diabrotica virgifera virgifera LeConte (Coleoptera: Chrysomelidae) was used to assess whether conditions of the natal field, subsequent laboratory rearing, or genetic population origin affect phenotypic traits of fitness, activity, or morphometrics. Standardized laboratory bioassays with large sample sizes revealed that none of the 16 tested traits, except crawling behaviours, appeared consistently stable across all seven tested colonies. Environmental conditions in the natal field of the $F_{0}$ generation affected trait averages of the subsequently reared $F_{1}$ generation in laboratory in $c a .47 \%$ of cases, and trait variability in $67 \%$ of cases. This was apparent for fitness and morphometrics, but less obvious for activity traits. Early generation laboratory rearing affected trait averages in ca. 56\% of cases: morphometrics changed; fecundity and egg survival increased from $F_{1}$ to $F_{2}$. Trait variability increased or decreased in $38 \%$ of cases. Laboratory rearing for over more than 190 generations affected the trait averages in $60 \%$ of cases, reflected by decreases in flight activity and increases in body size, weight, and fecundity to some extent. It had little effect on trait variability, especially so for morphometric variability. The genetic population origin affected average levels of $55 \%$ and variability of $63 \%$ of phenotypic traits. A comparison among D. v. virgifera studies might be difficult if they use different populations or laboratory colonies. It is advised to consider possible effects of original field conditions, laboratory rearing, and population genetics when planning comparative studies targeting fitness, activity, or morphometric questions regarding Diabrotica species.
\end{abstract}

Keywords: phenotypic traits, fitness, activity, morphometrics, generation number, western corn rootworm, Coleoptera, Chrysomelidae, Zea mays

(Accepted 21 October 2013; First published online 22 November 2013)

\footnotetext{
*Author for correspondence

Phone: +41324214882

Fax: +41324214871

E-mail: s.toepfer@cabi.org
}

\section{Introduction}

Measures of insect phenotypic traits are used to address a diverse range of basic and applied research questions. For example, phenotypic life history traits and their heritability are often investigated in theoretical insect ecology 
(e.g., Spurgeon, 2012) and evolutionary biology (e.g., Hill \& Caballero, 1992; Stearns, 1992; Miyatake \& Yamagishi, 1999; Khazaeli \& Curtsinger, 2010). Behavioural phenotypic traits of pest insects are specifically investigated to better understand questions in applied insect ecology (Huettel, 1976), usually with the goal of improving pest management strategies (Richerson \& Cameron, 1974; Prokopy et al., 1975).

Because of their relatively small size, ease of observation, and high rate of reproduction, many studies on insects are conducted under laboratory conditions. Laboratory studies can reduce environmental variation allowing researchers to focus on biological variation within and among populations. Often insects are easily mass-collected from the field and brought into the laboratory at different times for rearing specific colonies, which results in studies on specimens from colonies of varying origins, initial (=founder) population sizes, and generation numbers. A number of studies have shown that working in the laboratory either with fieldcollected individuals or with long-term mass-reared colonies can both have limitations (Richerson \& Cameron, 1974; Prokopy et al., 1975; Bush et al., 1976; Huettel, 1976; Boiler \& Chambers, 1977; Chambers, 1977). For example, they might differ in traits due to trans-generational environmental effects originating from the field conditions, also called parental effects from the field (Diamond et al., 2010) or due to geographical distances among populations of origin (Diamantidis et al., 2011). An advantage of using fieldcollected insects is that they have not been selected for adaptation to certain laboratory conditions (Rossler, 1975). An advantage of laboratory-reared insects is that those transgenerational effects can be standardized to laboratory conditions, i.e., laboratory rearing can eliminate differential responses specific to the environment in the different fields. But, this carries the risk of laboratory adaption, i.e., genetic changes in traits over generations may appear (Rossler, 1975; Miyatake \& Yamagishi, 1999; Scannapieco et al., 2009; Spurgeon, 2012).

One of the most economically important agricultural pest insects, the western corn rootworm, Diabrotica virgifera ssp. virgifera LeConte (Coleoptera: Chrysomelidae) is often studied in the field or using specimens that can be easily collected from the field. However, many studies use beetles from laboratoryreared colonies (Lefko et al., 2008).

Diabrotica $v$. virgifera is a univoltine maize pest, which overwinters as eggs in the soil (Chiang, 1973; Krysan \& Miller, 1986). After maize has germinated, the eggs hatch and the three larval instars feed almost exclusively on maize roots (Moeser \& Hibbard, 2005) often causing severe damage leading to plant lodging and extensive yield losses. Adults emerge around flowering stage of maize (Toepfer \& Kuhlmann, 2006), and can reduce crop yields through silk feeding, which interferes with maize pollination (Chiang, 1973; Culy et al., 1992).

Diabrotica $v$. virgifera is hypothesized to have originated in Mexico, where a number of pestiferous Diabrotica species occur (Branson \& Krysan, 1981; Krysan \& Smith, 1987). With the expansion of monoculture maize-growing in the second half of the 20th century, D. v. virgifera became a major pest of maize in the USA (Krysan \& Miller, 1986; Levine \& Oloumi-Sadeghi, 1991). Between the early 1980s and early 2000s, D. v. virgifera was accidentally introduced from North America into Europe on several occasions, leading to several independently expanding populations in Europe (Kiss et al., 2005; Miller et al., 2005; Ciosi et al., 2008).
Since its first detection as a maize pest in the USA in 1909 (Gillette, 1912) and in Europe in 1992 (Sivcev et al., 1994), numerous studies on its biology, behavioural ecology and control measures have been conducted. Indeed, nearly 900 papers have been published dealing with $D$. virgifera (search 'western corn rootworm' or 'Diabrotica virgifera' in publication titles found in CAB Abstracts (1900-2012)). Since the 1950s, the cumulative number of bioassays exceeded 150 published papers by 2012, primarily dealing with quantifying the life history traits, with impacts of weather conditions on different life stages, the host suitability of maize hybrids as well as of potential alternative host plants, and with the development of resistances to insecticides and transgenic traits in maize (Vidal et al., 2005). Standardized laboratory studies were possible due to laboratory-reared colonies of $D$. v. virgifera, such as those at the USDA-ARS North Central Agricultural Research Laboratory (NCARL; Brookings, South Dakota, USA). Another major advantage was the selection of a non-diapause colony of D. v. virgifera in the early 1970s at NCARL (Branson, 1976), which reduced generation time from many to a few months allowing four generations each year as opposed to the normal 1 year generation for wild type $D . v$. virgifera. Currently, a large proportion of laboratory studies are conducted using this nondiapause colony (Kim et al., 2007; Lefko et al., 2008; Meihls et al., 2008). However, reliance on a long-term laboratory colony raises concerns about whether this non-diapause colony, or any other laboratory colony of $D$. v. virgifera, still reflects 'natural' D. v. virgifera (Kim et al., 2007; Lefko et al., 2008). The non-diapause colony, for example, has been in culture for more than 190 generations without out-crossings or refreshments with field-collected beetles (Kim et al., 2007). Also, many of the available diapause colonies from different laboratories have been reared for more than ten generations. In contrast, the researchers that do not have access to constantly reared laboratory cultures establish short-term cultures for the duration of their usually 2-4-year-long projects, or conduct research on field-collected $F_{0}$ or on $F_{1}$ individuals.

However, most studies do not aim to compare different laboratory colonies or wild D. v. virgifera populations (Spencer et al., 2009). Thus, the effect of the original environmental conditions, laboratory rearing or genetic differentiation between populations has seldom been addressed. Lefko et al. (2008) compared some fitness traits among differently reared and crossed laboratory colonies of $D$. v. virgifera. They found that particularly in early generations (e.g., during rearing from $F_{0}$ to approximately $F_{4}$ or $F_{5}$ ) survival rates can vary widely between colonies and between generations. Kim et al. (2007) investigated the genetic variability of the USDA-ARS NCARL non-diapause colony $\left(>F_{190}\right)$, several diapause colonies initially collected from the USA $\left(>F_{22}, F_{3}\right.$ to $\left.F_{8}\right)$, as well as some wild USA populations. Astonishingly, the neutral genetic variability revealed by microsatellite genotyping was similar among most laboratory colonies and wild populations (Kim et al., 2007). A moderate loss in genetic variability $\left(\right.$ ca. $15-39 \%$,) was only found in the laboratory reared $\left(>F_{190}\right)$ non-diapause colony. In general, there was little evidence that the laboratory colonies underwent significant genetic bottlenecks or selection processes compared to wild populations. The results of Kim et al. (2007) suggest that the USA $D$. v. virgifera colonies maintained in laboratories are usually genetically similar to each other and to wild populations, at least for the neutral genetic variability that was studied. However, establishing a relationship between 
Table 1. Studied colonies of three D. v. virgifera populations reared at CABI, Delemont, Switzerland under identical laboratory condition; from the Central/Southeastern European population (CSE), from the Northwestern Italian population (NW I), and the Central-toNorthern US American population USA, The USA SD2 colony is a colony selected for non-diapause.

\begin{tabular}{|c|c|c|c|c|c|c|c|}
\hline Population & Central & Southeaster & European & Northwestern & Central-to-Nor & hern USA & \\
\hline $\begin{array}{l}\text { Tested colony } \\
\text { Colony code }\end{array}$ & $\begin{array}{l}\text { Tolna } \\
\text { county } \\
\text { CSE1 }\end{array}$ & $\begin{array}{c}\text { Timisoara } \\
\text { county } \\
\text { CSE2 }\end{array}$ & $\begin{array}{l}\text { Severnobacki } \\
\text { county } \\
\text { CSE3 }\end{array}$ & $\begin{array}{l}\text { Lombardy } \\
\text { province } \\
\text { NW I }\end{array}$ & $\begin{array}{l}\text { Pennsylvania } \\
\text { state } \\
\text { USA PA }\end{array}$ & $\begin{array}{l}\text { South Dakota } \\
\text { state } \\
\text { USA SD1 }\end{array}$ & $\begin{array}{l}\text { South Dakota } \\
\text { state } \\
\text { USA SD2 }\end{array}$ \\
\hline $\begin{array}{l}\text { Year of field collection } \\
\text { Tested generation } \\
n \text { pairs of adults tested }\end{array}$ & $\begin{array}{l}2006 \\
F_{1} \\
110\end{array}$ & $\begin{array}{l}2006 \\
F_{1} \\
98\end{array}$ & $\begin{array}{l}2006 \\
F_{2} \\
148\end{array}$ & $\begin{array}{l}2006 \\
F_{1}, F_{2} \\
142,38\end{array}$ & $\begin{array}{l}2000 \\
F_{8} \\
146\end{array}$ & $\begin{array}{l}1986 \\
F_{23} \\
150\end{array}$ & $\begin{array}{l}1966 \\
F_{191} \\
196\end{array}$ \\
\hline
\end{tabular}

neutral genetic variability, which might be less subject to natural selection, and phenotypic variability, which primarily results from the pressures of natural selection, remains difficult to establish. Therefore, it is largely unknown whether laboratory-reared colonies also have similar comparable phenotypes. It has been suggested that the non-diapause beetles from NCARL appear somewhat larger and less active than beetles from other colonies ( $\mathrm{Ch}$. Nielson, Brookings, SD, USA, 2007 pers. comm.; K. Gloyna, Sagerheide, Germany, 2008 pers. comm.). In contrast to laboratory colonies, it is wellreported that field-collected $F_{0} D$. $v$. virgifera adults vary in their fitness (Spencer et al., 2009), depending on environmental conditions or insect age at collection (Li et al., 2010), nutritional value of the maize plants, and on many other factors. Whether effects of the previous field environment can still be detected as parental effects in laboratory colonies during subsequent rearing (Bernardo, 1996; Mousseau \& Fox, 1998; Johnston et al., 2004) is unknown for D. v. virgifera.

Our study used several laboratory colonies with the goal to rear, observe, and measure hypothesized differences in phenotypic traits using standardized bioassays from Li et al. (2009) and Toepfer et al. (2012). $F_{1}$ generations from the Central/Southeastern European D. v. virgifera population, but from different locations that present no neutral genetic differentiation (Miller et al., 2006, 2007, Ciosi et al., 2008) were used to investigate hypothesized parental effects originating from the field situation. An $F_{1}$ and an $F_{2}$ generation of laboratoryreared Northwest Italian $D$. v. virgifera were compared to evaluate whether researchers can reliably use $F_{1}$ generations or should rather rear D. v. virgifera at least up to the $F_{2}$ generation before starting bioassays. Furthermore, D. v. virgifera from different generations of three long-reared USA colonies were used to study hypothesized changes in phenotype over the time of rearing. Finally, colonies from genetically distinct populations (two independently founded European outbreaks and one USA population) were compared to estimate the hypothesized effect of neutral genetic differentiation between populations of origin on phenotype. This will help researchers decide whether to work with laboratory colonies or with fieldcollected insects in comparative studies targeting fitness, activity, or morphometric questions regarding Diabrotica pest species.

\section{Materials and methods}

\section{D. v. virgifera colonies and standard rearing conditions}

Experiments were conducted with seven laboratory-reared colonies. They originated from three genetically distinct populations (here synonym for genotypic classes, Johnston et al., 2004) (table 1): the Central/Southeastern European population (CSE European), the Northwest Italian population (NW INW I) as defined by Miller et al. (2005), and the USA population (excluding Texas) as defined by Miller et al. (2006, 2009) (table 1). The neutral genetics of the CSE population were characterized by about 3.4 different alleles directly counted per locus $(D C)$, and an expected heterozygosity $\left(H_{e}\right)$ of about 0.45 under Hardy-Weinberg assumptions (Miller et al., 2006, 2007; Ciosi et al., 2008). The DC of the NW Italian population was about 3.3 and the $H_{e}$ about 0.35 (Miller et al., 2005, 2006, 2009; Ciosi et al., 2008). The USA population was characterized by DCs from 5.3 to 9.0 , allele richness $A R$ from 5.3 to $8.9, H_{e}$ from 0.59 to 0.69 , and the observed heterozygosity $H_{o}$ from about 0.59 to 0.76 (Kim \& Sappington, 2005; Miller et al., 2005, 2006; Kim et al., 2007; Ciosi et al., 2008). Within each genetic population (CSE European, NW Italian, USA), genetic variation ranged from none to very little (Kim \& Sappington, 2005; Miller et al., 2005, 2006; Kim et al., 2007; Ciosi et al., 2008).

Three CSE European population colonies were each founded with more than 1000 adults $\left(F_{0}\right)$ that were collected from heavily infested maize fields in Tolna County of southern Hungary (colony CSE1), Timisoara County of Northern Romania (colony CSE2), and Severnobacki County of Northern Serbia (colony CSE3). A single colony originated from the NW Italian population; it was established from 500 adults $\left(F_{0}\right)$ collected from an infested maize field in Lombardy County (colony NW I). Three D. v. virgifera colonies originated from the USA; they were established from at least 1000 adults $\left(F_{0}\right)$ collected from fields in Pennsylvania or South Dakota (colonies USA PA, USA SD1, and USA SD2). For dates of colony establishments refer to table 1 . The number of field collect founders of each colony or population was large enough to avoid random genetic drift and inbreeding depression, according to Miyatake \& Yamagishi (1999).

Before experiments, each D. v. virgifera colony was reared at CABI, Delemont, Switzerland under identical laboratory conditions, as per George \& Ortman (1965), Branson et al. (1975), and Li et al. (2009). Eggs were incubated at a temperature of $25^{\circ} \mathrm{C}$ (during photophase: L) and $21^{\circ} \mathrm{C}$ (during scotophase: D) for 14-24 days to initiate egg hatching. About 2000-3000 untreated seeds of the maize hybrid Gavott (UFA Semences, Bussigny, Switzerland) were soaked in water for $24 \mathrm{~h}$ and germinated in a plastic tray $(\mathrm{l}: 330 \mathrm{~mm}$, w: $190 \mathrm{~mm}$, and $\mathrm{h}: 110 \mathrm{~mm}$ ) with a gauze lid. Two to three days after germination, ready-to-hatch eggs were transferred in their overwintering soil onto a filter paper and then onto the seeds 
in the plastic trays (approx. 5000 eggs per tray) and maintained at a temperature of L: D, $25^{\circ} \mathrm{C}: 21^{\circ} \mathrm{C}$, and light regime of L: D, 14: 10. Emerging larvae found unlimited food (maize roots). After 14-20 days, third instar larvae were transferred along with the maize seedlings into gauze-covered cylinders (dia.: $120 \mathrm{~mm}$, h: $140 \mathrm{~mm}$ ) containing sterilized field soil for pupation (soil sieved at $<5 \mathrm{~mm}$ mesh size, $30 \%$ moisture). The transferred maize continued to provide food for the developing larvae until they pupated. Emerging adults were collected and either used for experiments (see below) or transferred to gauze cages $(450 \times 450 \times 600 \mathrm{~mm})$ for further mass rearing of subsequent generations according to Krysan \& Miller (1986) and Singh \& Moore (1999) (temperature L: D, $24-26^{\circ} \mathrm{C}$ : $18-20^{\circ} \mathrm{C}, 40-60 \%$ relative humidity, light regime L: D, 14: 10). For mass rearing, at least 100 mated females of each colony and rearing cycle were allowed to lay eggs into petri dishes with moist, sterilized and sieved black Chernozem field soil ( $<200 \mu \mathrm{m}$ mesh size) up to 2 months after collection. The petri dishes were changed every 2 weeks. The soil with eggs was washed through a $0.25 \mathrm{~mm}$ sieve with room temperature tap water. The number of recovered eggs on the sieve was estimated. Eggs were then stored in the sterile moist soil and kept at a temperature of L: D, 25: $21^{\circ} \mathrm{C}$ during 2 weeks for pre-diapause (Krysan, 1972; Branson, 1976). Eggs were then overwintered for diapause at $6-8^{\circ} \mathrm{C}$ for 5-7 months (Krysan, 1982), except for eggs from the nondiapause USA colony (USA SD2), which hatch in about 14 days, and were used for experiments or further mass rearing immediately.

\section{Assessing phenotypic traits}

Sixteen phenotypic traits were assessed according to Li et al. (2009, 2010): five fitness traits (fecundity, overwintering egg survival, larva-to-adult survival, egg-to-adult survival, and adult lifespan), four activity traits (proportion of adults crawling, crawling speed, proportion of adults flying, and flight take-off response), and seven morphometric traits of adults (fresh body weight, elytra length and width, pronotum length and width, head capsule width, and hind tibia length). Assessments were conducted with D. v. virgifera from two rearing series (= repetitions) of each colony (Begley, 2013). Data from two series were pooled per colony as traits rarely showed any difference between series within a generation. Assessments of adult D. $v$. virgifera were only conducted with young 1-6 day old individuals to reduce the influence of age and the rearing environment on shaping the phenotypic traits as investigated by Li et al., $(2009,2010)$. Moreover, D. v. virgifera individuals develop and mature at different speed adding to an increasing variability in trait data with age of a colony, which renders comparisons between colonies or populations difficult (Li et al., 2009, 2010). Averages, ranges, variation, and statistical tests of all trait data are presented in tables $2-5$ to allow cross-checking, and the repetition of experiments by users according to Begley (2013).

\section{Fitness traits}

In the laboratory, newly emerged adults were sexed according to antenna length (Staetz et al., 1976; Kuhar \& Youngman, 1995) and tarsal characteristics (Hammack \& French, 2007). Male-female pairs were transferred to small bioassay containers (2 daylight simulating Osram HQI-BT 400 watt lamps at light regime L: D, 14: 10; temperature L: D, 24: $18^{\circ} \mathrm{C}$; relative humidity $60 \%$ ). The containers consisted of two plastic urinalysis cups (dia.: $48 \mathrm{~mm}$, h: $80 \mathrm{~mm}$ ), stacked one inside the other providing approximately $175 \mathrm{~cm}^{3}$ of space (for details see Li et al., 2009; Toepfer et al., 2012). The upper cup had a $10-\mathrm{mm}$ hole in the bottom to give the female access to the lower, soil-filled cup for egg-laying. Abundant food was provided in each container (two soft, unripe kernels of organically-produced maize one $13 \times 13 \times 13 \mathrm{~mm}$ piece of zucchini flesh, one $13 \times 13 \times 13 \mathrm{~mm}$ piece of pumpkin flesh (Li et al., 2009), and a $5 \times 5 \times 5 \mathrm{~mm}$ piece of artificial pollen diet (Branson \& Jackson, 1988; Singh \& Moore, 1999). A $10 \times 5 \times 5 \mathrm{~mm}$ cube of $15 \%$ water agar served as a water source for the adults. Food and agar were changed every 5-7 days. Each tested pair of $D$. v. virgifera was provided with the same amount of food, as D. v. virgifera fitness and activity is known to be influenced by recent diet experience (Levine et al., 2002; Mabry et al., 2004).

To assess adult lifespan, bioassay containers were checked daily for live and dead adults. The date of death was recorded, lifespan was calculated, and dead adults were removed and not replaced. The latter was implemented, because $D$. $v$. virgifera females are known to be able to lay all their eggs after one mating only (Spencer et al., 2009) and because survival curves of males and females are similar (Li et al., 2009, 2010). The lifespan assessment was stopped after 70 days because this period is long enough to reliably reflect the total adult lifespan (Li et al., 2009). The proportion of females and males surviving until day 70 was calculated for each tested colony.

To assess fecundity, two teaspoons of moist, sterile black Chernozem field soil (sieved at $0.15 \mathrm{~mm}$ mesh size; $25-35 \mathrm{wt} \%$ moisture) were placed into the lower cup of the bioassay containers (see above) after 7 days of maturation (Branson \& Johnson, 1973; Hill, 1975). Moisture remained stable due to nearly-closed environment of the double-stacked bioassay container. Then, every 14 days, the lower cup of the bioassay container (containing soil and eggs) was removed and replaced with a new one. The soil with eggs was washed with room temperature tap water through a $0.25 \mathrm{~mm}$ sieve, and the recovered eggs were counted. The accumulated 98-day (=total) realized fecundity was calculated per individual female for a number of colonies, but then reduced to measuring the 70-day fecundity as Li et al. (2009) reported a linear relationship between fecundity and age, and suggested that the 70-day fecundity is enough to reliably estimate and compare colony fecundities. Eggs were then stored in sterile moist soil and kept as described above.

To determine the overwintering survival of eggs, the soil with eggs was washed through a $0.25 \mathrm{~mm}$ sieve after 5 months of diapause. Egg survival was checked according to Modic et al. (2008) under a stereomicroscope and recorded per individual parental female of each tested colony.

To determine larva-to-adult survival, 200 successfully overwintered viable eggs were incubated at a temperature of L: D, $25: 21^{\circ} \mathrm{C}$ for $14-24$ days to initiate egg hatching. Because not every female had laid enough eggs to obtain 200 eggs after overwintering, this portion of the study was conducted with pooled egg batches. The larvae and pupae were reared as described above. Adult emergence was recorded daily until there were four consecutive days without any emergence. The total number of emerged adults was divided by the initial number of viable eggs $(n=200)$ to calculate the hatchedlarva-to-adult survival. 
Table 2. Differences between $F_{1}$ colonies from different locations of the same genetic population (field influenced parental effects) assessed through average levels and variability of phenotypic traits between $F_{1}$ from Southern Hungary (colony CSE1), and from northern Romania (colony CSE2) (both Central/Southeastern European genetic population CSE). Different letters in rows indicate significant differences of mean values (small fonts) or variances (capital fonts) of traits between colonies according to fdr correction; $n=$ number of individuals, $\mathrm{SD}=$ standard deviation, $C V=$ coefficient of variation. [Note: 98 day - fecundity for CSE1: $461 \pm 398$ eggs with range of $0-1386(n=55)$ and CV of 0.86 versus CSE 2: $196 \pm 226$ eggs with range of $0-856(n=48)$ and CV of $1.15(\mathrm{P}<0.005$ between means and between variances $)$.

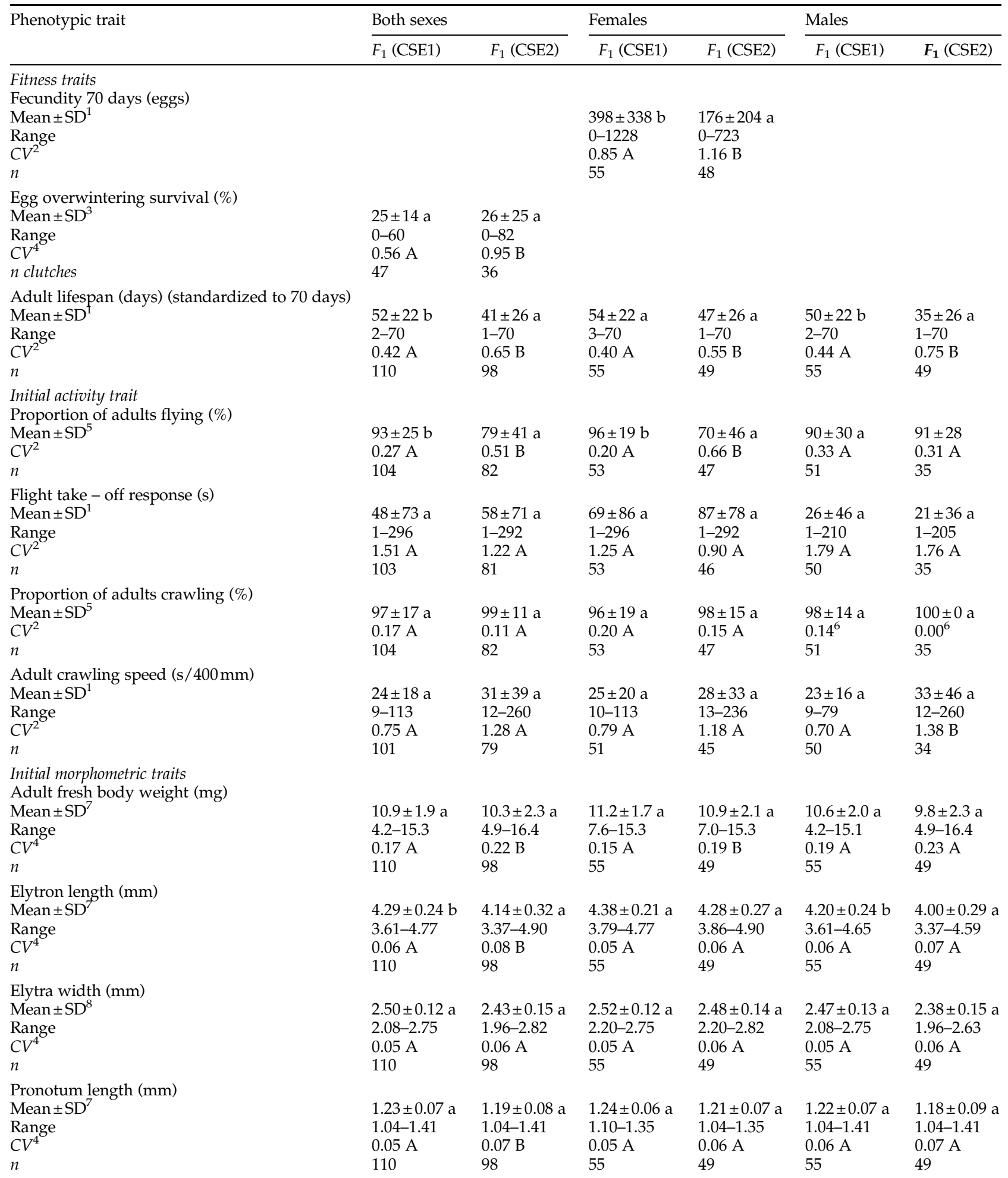


Table 2. (Cont.)

\begin{tabular}{|c|c|c|c|c|c|c|}
\hline \multirow[t]{2}{*}{ Phenotypic trait } & \multicolumn{2}{|l|}{ Both sexes } & \multicolumn{2}{|l|}{ Females } & \multicolumn{2}{|l|}{ Males } \\
\hline & $F_{1}(\mathrm{CSE} 1)$ & $F_{1}(\mathrm{CSE} 2)$ & $F_{1}(\mathrm{CSE} 1)$ & $F_{1}(\mathrm{CSE} 2)$ & $F_{1}(\mathrm{CSE} 1)$ & $\boldsymbol{F}_{\mathbf{1}}(\mathrm{CSE} 2)$ \\
\hline \multicolumn{7}{|c|}{ Pronotum width (mm) } \\
\hline Range & $1.29-1.65$ & $1.22-1.71$ & $1.35-1.65$ & $1.35-1.71$ & $1.29-1.65$ & $1.22-1.65$ \\
\hline$C V^{4}$ & $0.05 \mathrm{~A}$ & $0.08 \mathrm{~B}$ & $0.05 \mathrm{~A}$ & $0.06 \mathrm{~B}$ & $0.05 \mathrm{~A}$ & $0.08 \mathrm{~B}$ \\
\hline$n$ & 110 & 98 & 55 & 49 & 55 & 49 \\
\hline Range & $1.10-1.35$ & $0.98-1.35$ & $1.10-1.35$ & $1.04-1.35$ & $1.10-1.29$ & $0.98-1.29$ \\
\hline$C V^{4}$ & $0.05 \mathrm{~A}$ & $0.06 \mathrm{~A}$ & $0.05 \mathrm{~A}$ & $0.06 \mathrm{~A}$ & $0.05 \mathrm{~A}$ & $0.07 \mathrm{~A}$ \\
\hline$n$ & 110 & 98 & 55 & 49 & 55 & 49 \\
\hline \multicolumn{7}{|c|}{ Hind tibia length (mm) } \\
\hline Mean $\pm \mathrm{SD}^{7}$ & $1.80 \pm 0.10 \mathrm{~b}$ & $1.74 \pm 0.11 \mathrm{a}$ & $1.81 \pm 0.11 \mathrm{~b}$ & $1.76 \pm 0.12 \mathrm{a}$ & $1.79 \pm 0.09 \mathrm{~b}$ & $1.72 \pm 0.11$ \\
\hline Range & $1.50-2.00$ & $1.30-2.00$ & $1.50-2.00$ & $1.50-2.00$ & $1.60-2.00$ & $1.30-1.90$ \\
\hline \multicolumn{7}{|c|}{ Overall phenotypic $C V$} \\
\hline Mean \pm SD $^{6}$ & $0.38 \pm 0.46 \mathrm{~A}$ & $0.49 \pm 0.52 \mathrm{~B}$ & $0.36 \pm 0.41 \mathrm{~A}$ & $0.44 \pm 0.47 \mathrm{~B}$ & $0.34 \pm 0.55 \mathrm{~A}$ & $0.46 \pm 0.63 \mathrm{~B}$ \\
\hline
\end{tabular}

${ }^{1} \mathrm{fdr}$-corrected multiple pairwise contrast comparisons with sequential Sidak procedure after GZLM for non-normally distributed data. Distribution considered as Poisson log-linear link function.

2 Difference in variation through comparing variances using Levine's tests in case of non-normally distributed data.

3 No comparison because of only few data values for each colony due to pooled rearing of larvae.

${ }^{4}$ Difference in variation through comparing variances using F - statistics in case of normally distributed data (with or without transformation).

${ }^{5}$ fdr-corrected multiple pairwise contrast comparisons with sequential Sidak procedure after GZLM because of non-normally distributed data. Distribution considered as binary probit link function.

6 Differences of overall $C V$ according to non-parametric related samples sign test

7 fdr-corrected multiple Games-Howell pairwise comparison post hoc test after ANCOVA because of close-to-normally distributed data (with or without transformation) and unequal homogeneity of variances.

${ }^{8}$ fdr-corrected multiple comparison Tukey post hoc range test after ANCOVA because of close-to-normally distributed data (with or without transformation) and equal homogeneity of variances.

Egg-to-adult survival was calculated by combining the data from the overwintering survival of eggs with the survival from hatched larvae via pupae until adulthood.

\section{Initial activity traits}

Crawling activity of young adults was assessed as a measure of short distance within-field dispersal; and flight activity as a measure of dispersal capability in general. Crawling speed and the proportion of adults crawling were measured using the methodology of Mabry et al. (2004) and Li et al. (2010). Flight take-off response and the proportion of adults flying were measured as by Duan et al., (1998); Toepfer et al., (2005); and Li et al., (2010). Measurements were made using young adults (6 day old=initial activity trait), because activity measures on young adults better reflect activity differences between individuals or colonies than measures on mature adults which are much influenced by nutritional status and egg load (Li et al., 2010). The trials were conducted under laboratory conditions of $24^{\circ} \mathrm{C}, 50-60 \%$ relative humidity, and simulated daylight (2 Osram HQI-BT $400 \mathrm{~W}$ daylight lamps). They were always conducted between 14:00 and 16:00 as both temperature (VanWoerkom et al., 1980), and time of the day (Coats et al., 1986) can influence activity.

As for crawling, a 400-mm long crawling section was marked on a $700-\mathrm{mm}$ long transparent plastic tube of $9 \mathrm{~mm}$ diameter. The plastic tube was vertically fixed to a ring stand. A plastic vial containing an adult was slipped under the open bottom end of the tube allowing the adult to crawl into the tube and upwards. Once the adult reached the $400 \mathrm{~mm}$ section, the time from the starting to the end point was recorded as well as the incidence of crawling, not crawling or crawling but not finishing the $400 \mathrm{~mm}$ distance within $300 \mathrm{~s}$. The trial ended when the adult had crawled the $400 \mathrm{~mm}$ or when $300 \mathrm{~s}$ had elapsed (according to Li et al., 2010). Tested adults were returned to the rearing containers. The proportion of adults crawling, and their mean crawling speed were calculated for young adult males and females by colony. As crawling and flight activity appeared highly correlated (Li et al., 2010), the crawling trait measure was skipped in later colony assessments.

To assess flight activity, flight stands were used that consisted of a wooden pin (h: $40 \mathrm{~mm}$, dia.: $10 \mathrm{~mm}$ ) fixed onto the end of an inverted white plastic funnel (h: $160 \mathrm{~mm}$, dia.: $135 \mathrm{~mm}$ at base). The base of the funnel was surrounded with water to prevent the adults from walking off of the stand. An individual was gently released onto the base of the funnel using an aspirator. Following release, the incidence of take-off and the time between release and take-off were recorded. A trial ended when the adults flew off the stand or when $300 \mathrm{~s}$ had elapsed without take-off (Toepfer et al., 2005; Li et al., 2010). Tested insects were returned to their bioassay containers. The proportion of adults flying and the mean time 
Table 3. Differences between the $F_{1}$ and $F_{2}$ generation of the same genetic population (effects of early generation laboratory rearing), assessed through average levels and variability of phenotypic traits between a laboratory-reared $F_{1}$ and $F_{2}$ of $D$. $v$. virgifera originating from a field-collected $F_{0}$ of the Northwestern Italian genetic population (NW I). Different letters in rows indicate significant differences of mean values (small fonts) or variances (capital fonts) of traits between colonies according to fdr correction; $n=$ number of individuals, $\mathrm{SD}=$ standard deviation, $\mathrm{CV}=$ coefficient of variation.

\begin{tabular}{|c|c|c|c|c|c|c|}
\hline \multirow[t]{2}{*}{ Phenotypic trait } & \multicolumn{2}{|l|}{ Both sexes } & \multicolumn{2}{|l|}{ Females } & \multicolumn{2}{|l|}{ Males } \\
\hline & $F_{1}(\mathrm{NW} \mathrm{I})$ & $F_{2}(\mathrm{NW} \mathrm{I})$ & $F_{1}(\mathrm{NW} \mathrm{I})$ & $F_{2}(\mathrm{NW} \mathrm{I})$ & $F_{1}(\mathrm{NW} \mathrm{I})$ & $F_{2}(\mathrm{NW} \mathrm{I})$ \\
\hline \multicolumn{7}{|c|}{ Fitness traits } \\
\hline Range & & & $0-1327$ & $0-1323$ & & \\
\hline$C V^{2}$ & & & $0.65 \mathrm{~A}$ & $0.59 \mathrm{~A}$ & & \\
\hline$n$ & & & 71 & 19 & & \\
\hline Range & $0-60$ & $51-90$ & & & & \\
\hline$C V^{4}$ & $0.60 \mathrm{~B}$ & $0.17 \mathrm{~A}$ & & & & \\
\hline$n$ clutches & 64 & 16 & & & & \\
\hline \multicolumn{7}{|c|}{ Hatched-larva-to-adult survival (\%) } \\
\hline Mean \pm SD $^{5}$ & $34 \pm 4$ & $10 \pm 0$ & & & & \\
\hline Range & 3-39 & $10-10$ & & & & \\
\hline$C V^{6}$ & 0.13 & 0.00 & & & & \\
\hline$n$ clutches & 64 & 16 & & & & \\
\hline \multicolumn{7}{|c|}{ Egg-to-adult survival (\%) } \\
\hline$n$ clutches & 64 & 16 & & & & \\
\hline \multicolumn{7}{|c|}{ Adult lifespan (days) (standardized to 70 days) } \\
\hline Mean $\pm \mathrm{SD}^{1}$ & $59 \pm 19$ a & $62 \pm 18$ a & $57 \pm 19 a$ & $68 \pm 5 b$ & $60 \pm 19 a$ & $57 \pm 24 \mathrm{a}$ \\
\hline Range & $3-70$ & $3-70$ & $7-70$ & $55-70$ & $3-70$ & $3-70$ \\
\hline$C V^{2}$ & $0.33 \mathrm{~A}$ & $0.29 \mathrm{~A}$ & $0.34 \mathrm{~B}$ & $0.07 \mathrm{~A}$ & $0.32 \mathrm{~A}$ & $0.43 \mathrm{~A}$ \\
\hline$n$ & 142 & 38 & 71 & 19 & 71 & 19 \\
\hline \\
\hline \multicolumn{7}{|c|}{ Proportion of adults flying (\%) } \\
\hline Mean $\pm \mathrm{SD}^{7}$ & $99 \pm 8$ a & $94 \pm 23$ a & $99 \pm 12 \mathrm{a}$ & $89 \pm 32$ a & $100 \pm 0 \mathrm{a}$ & $100 \pm 0 \mathrm{a}$ \\
\hline$C V^{2}$ & $0.09 \mathrm{~A}$ & $0.25 \mathrm{~B}$ & $0.12 \mathrm{~A}$ & $0.35 \mathrm{~B}$ & $0.0 \mathrm{~A}$ & $0.0 \mathrm{~A}$ \\
\hline$n$ & 140 & 36 & 70 & 19 & 70 & 17 \\
\hline \multicolumn{7}{|c|}{ Flight take-off response (s) } \\
\hline Mean $\pm \mathrm{SD}^{1}$ & $20 \pm 36$ a & $17 \pm 26 \mathrm{a}$ & $29 \pm 43 \mathrm{a}$ & $28 \pm 31 \mathrm{a}$ & $12 \pm 25 \mathrm{a}$ & $5 \pm 7 \mathrm{a}$ \\
\hline Range & $1-247$ & $1-112$ & $1-247$ & $2-112$ & $1-176$ & $1-28$ \\
\hline Mean \pm SD $^{8}$ & $4.15 \pm 0.29 \mathrm{a}$ & $4.21 \pm 0.29 b$ & $4.26 \pm 0.26 \mathrm{a}$ & $4.32 \pm 0.32 \mathrm{a}$ & $4.04 \pm 0.28 \mathrm{a}$ & $4.11 \pm 0.22 \mathrm{~b}$ \\
\hline Range & $2.45-4.77$ & $3.67-4.84$ & $3.49-4.77$ & $3.79-4.84$ & $2.45-4.47$ & $3.67-4.41$ \\
\hline$C V^{4}$ & $0.07 \mathrm{~A}$ & $0.07 \mathrm{~A}$ & $0.06 \mathrm{~A}$ & $0.08 \mathrm{~B}$ & $0.07 \mathrm{~A}$ & $0.05 \mathrm{~A}$ \\
\hline$n$ & 142 & 38 & 71 & 19 & 71 & 19 \\
\hline \multicolumn{7}{|l|}{ Elytra width (mm) } \\
\hline Mean $\pm \mathrm{SD}^{8}$ & $2.38 \pm 0.16 \mathrm{a}$ & $2.42 \pm 0.17 \mathrm{~b}$ & $2.44 \pm 0.14 \mathrm{a}$ & $2.46 \pm 0.20 \mathrm{a}$ & $2.32 \pm 0.14 \mathrm{a}$ & $2.38 \pm 0.14 b$ \\
\hline Range & $1.90-2.69$ & $1.96-2.75$ & $1.96-2.69$ & $2.08-2.75$ & $1.90-2.63$ & $1.96-2.63$ \\
\hline $\mathrm{CV}^{4}$ & $0.07 \mathrm{~A}$ & $0.07 \mathrm{~A}$ & $0.06 \mathrm{~A}$ & $0.08 \mathrm{~B}$ & $0.06 \mathrm{~A}$ & $0.06 \mathrm{~A}$ \\
\hline$n$ & 142 & 38 & 71 & 19 & 71 & 19 \\
\hline \multicolumn{7}{|c|}{ Overall phenotypic $C V$} \\
\hline Mean \pm SD $^{9}$ & $0.52 \pm 0.60 \mathrm{~A}$ & $0.42 \pm 0.51 \mathrm{~A}$ & $0.48 \pm 0.51 \mathrm{~A}$ & $0.33 \pm 0.39 \mathrm{~A}$ & $0.54 \pm 0.89 \mathrm{~A}$ & $0.43 \pm 0.56 \mathrm{~A}$ \\
\hline
\end{tabular}

${ }^{1}$ fdr-corrected multiple pairwise contrast comparisons with sequential Sidak procedure after GZLM for non-normally distributed data. Distribution considered as Poisson log-linear link function.

2 Difference in variation through comparing variances using Levine's tests in case of non-normally distributed data.

${ }^{3}$ fdr-corrected multiple Games-Howell pairwise comparison post hoc test after ANCOVA because of close-to-normally distributed data (with or without transformation) and unequal homogeneity of variances.

${ }^{4}$ Difference in variation through comparing variances using F-statistics in case of normally distributed data (with or without transformation).

${ }^{5}$ No comparison because of only few data values for each colony due to pooled rearing of larvae.

6 Differences in variances not tested because of lack of enough spread/level pairs.

${ }^{7}$ fdr-corrected multiple pairwise contrast comparisons with sequential Sidak procedure after GZLM because of non-normally distributed data. Distribution considered as binary probit link function.

${ }_{8}^{8}$ fdr-corrected multiple comparison Tukey post hoc range test after ANCOVA because of close-to-normally distributed data (with or without transformation) and equal homogeneity of variances.

${ }_{9}$ Differences of overall $\mathrm{CV}$ according to non-parametric related samples sign test. 


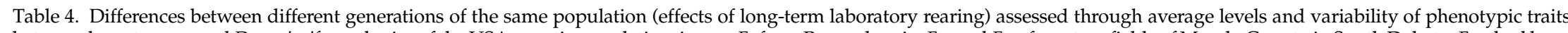

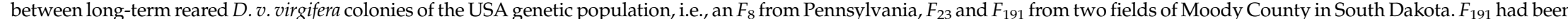

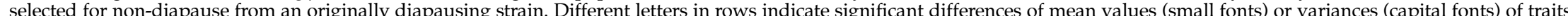

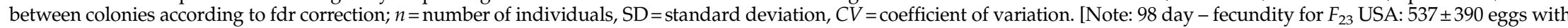
range of $0-1438(n=73)$ and CV of 0.73 versus $F_{191}$ USA: $703 \pm 488$ eggs with range of $0-1814(n=75)$ and CV of $0.69(P<0.005$ between means and $P>0.005$ between variances $)$.

\begin{tabular}{|c|c|c|c|c|c|c|c|c|}
\hline \multirow[t]{2}{*}{ Phenotypic trait } & \multicolumn{2}{|l|}{ Both sexes } & \multicolumn{3}{|l|}{ Females } & \multicolumn{3}{|l|}{ Males } \\
\hline & $F_{23}(\mathrm{USA}$ SD1) & $F_{191}(\mathrm{USA}$ SD2) & $F_{8}$ (USA PA) & $F_{23}$ (USA SD1) & $F_{191}$ (USA SD2) & $F_{8}$ (USA PA) & $F_{23}(\mathrm{USA}$ SD1) & $F_{191}$ (USA SD2) \\
\hline
\end{tabular}

\section{Fitness traits}

Fecundity 70 days (eggs)

Mean \pm SD $^{1}$

Range

$C V^{2}$

$\mathrm{CV}$

Egg overwintering survival (\%)

$\begin{array}{llll}\text { Mean } \pm \mathrm{SD}^{3} & 43 \pm 27 \mathrm{~b} & 18 \pm 13 \mathrm{a} & 24 \pm 17 \mathrm{ab} \\ \text { Range } & 0-100 & 0-56 & 0-78 \\ C V^{4} & 0.63 \mathrm{~A} & 0.71 \mathrm{~B} & 0.71 \mathrm{~B} \\ n \text { clutches } & 71 & 62 & 69\end{array}$

Adult lifespan (days) (standardized to 70 days)

Mean $+S^{1} \quad 59 \pm 18$ b $51 \pm 25$ a

$\begin{array}{lll}\text { Range } & 3-70 & 1-70\end{array}$

$\mathrm{CV}^{2} \quad 0.30 \mathrm{~A} \quad 0.49 \mathrm{~B}$

$n$

$146-150$

$2-70$
$0.37 \mathrm{AB}$

Initial activity trait

$150-196$

$\begin{array}{lll}58 \pm 16 \text { a } & 52 \pm 23 \mathrm{a} & 57 \pm 20 \mathrm{a} \\ 3-70 & 1-70 & 3-70 \\ 0.28 \mathrm{~A} & 0.45 \mathrm{~B} & 0.35 \mathrm{AB} \\ 73 & 75 & 99\end{array}$

Proportion of adults flying (\%)

Mean $\pm \mathrm{SD}^{5} \quad 82 \pm 39$

$\mathrm{CV}$

$0.48 \mathrm{~A}$

$67 \pm 47 \mathrm{~b}$

$0.71 \mathrm{~B}$

138

\section{$48 \pm 50$ a \\ $1.05 \mathrm{~B}$}

189

$71 \pm 46 \mathrm{C}$

$0.65 \mathrm{~A}$

Flight take - off response (seconds)

$\begin{array}{lll}\text { Mean } \pm \mathrm{SD}^{1} & 49 \pm 79 \mathrm{a} & 65 \pm 80 \mathrm{ab} \\ \text { Range } & 1-299 & 1-288 \\ C V^{2} & 1.63 \mathrm{~B} & 1.22 \mathrm{~A} \\ n & 139 & 133\end{array}$

$75 \pm 91 \mathrm{~b}$

$1-300$

$1.22 \mathrm{~A}$

182

$\begin{array}{lll}691 \pm 396 \mathrm{~b} & 481 \pm 353 \mathrm{a} & 613 \pm 408 \mathrm{ab} \\ 0-1489 & 0-1438 & 0-1403 \\ 0.57 \mathrm{~A} & 0.73 \mathrm{~A} & 0.67 \mathrm{~A} \\ 73 & 73 & 75\end{array}$

Proportion of adults crawling (\%)

Mean $\pm \mathrm{SD}^{5}$

133

$\begin{array}{ll}99 \pm 12 \mathrm{a} & 100 \pm 0 \mathrm{a} \\ 0.12 & 0.0\end{array}$

$n$

0.12

Adult crawling speed (seconds $/ 400 \mathrm{~mm}$ )

Mean $\pm \mathrm{SD}^{1}$

Range

$\mathrm{CV}^{2}$

$31 \pm 31$

0.0

189

$n$

$0.98 \mathrm{~A} \quad 0.93 \mathrm{~A}$

Initial morphometric traits

Adult fresh body weight $(\mathrm{mg})$

$\begin{array}{lll}\text { Mean } \pm \mathrm{SD}^{7} & 10.9 \pm 2.0 \mathrm{~b} & 9.4 \pm 1.8 \mathrm{a} \\ \text { Range } & 6.3-20.0 & 3.4-18.8 \\ \mathrm{C} V^{4} & 0.19 \mathrm{~A} & 0.20 \mathrm{~A}\end{array}$

n

146

149

$11.7 \pm 2.2 \mathrm{c}$
$6.4-18.0$
$0.19 \mathrm{~A}$
$8.0-20.0$

196

$0.17 \mathrm{~A}$

$51 \pm 77 \mathrm{a}$
$1-297$
$1.50 \mathrm{~A}$
71

$51 \pm 50 \mathrm{~b}$ $0.98 \mathrm{~B}$

72

$29 \pm 46$ a
1.58 B
97

$83 \pm 90$

$1-288$

$1.08 \mathrm{~A}$

67

$56 \pm 88$ a
$1-297$
$1.58 \mathrm{~A}$
92

$99 \pm 12$ a $\quad 100 \pm 0$ a

0.12

0.0

72

$\begin{array}{ll}38 \pm 40 \mathrm{a} & 34 \pm 23 \mathrm{a} \\ 13-271 & 14-163 \\ 1.05 \mathrm{~B} & 0.69 \mathrm{~A}\end{array}$

70

0.69
94

$\begin{array}{lll}60 \pm 19 \mathrm{~b} & 51 \pm 26 \mathrm{a} & 56 \pm 21 \mathrm{ab} \\ 3-70 & 2-70 & 2-70 \\ 0.32 \mathrm{~A} & 0.52 \mathrm{~B} & 0.39 \mathrm{AB} \\ 73 & 75 & 98\end{array}$

$93 \pm 26 \mathrm{~b}$

$0.28 \mathrm{~A}$

$83 \pm 38$ ab

$0.45 \mathrm{~B}$

$67 \pm 47$ a
$0.70 \mathrm{C}$

66

92

$46 \pm 82 \mathrm{a}$
$1-299$
$1.79 \mathrm{~A}$

$47 \pm 63$ a
$1-251$
$1.33 \mathrm{~A}$

$94 \pm 91 \mathrm{~b}$

$1.33 \mathrm{~A}$

$1-300$

66

$0.96 \mathrm{~A}$

$\begin{array}{ll}98 \pm 12 \mathrm{a} & 100 \pm 0 \mathrm{a} \\ 0.12 & 0.0\end{array}$

0.12

0.0
92

$\begin{array}{ll}24 \pm 11 \mathrm{a} & 36 \pm 40 \mathrm{~b} \\ 12-58 & 12-280 \\ 0.46 \mathrm{~A} & 1.11 \mathrm{~B} \\ 64 & 90\end{array}$

$9.9 \pm 1.9$ a

6.3-18.8

$0.19 \mathrm{~A}$

75

$12.0 \pm 2.1 \mathrm{~b}$
$7.3-18.0$
$0.17 \mathrm{~A}$
98

$10.1 \pm 1.8$

$6.3-15.5$

$8.8 \pm 1.6$

3.4-12.5

$0.18 \mathrm{~A}$

$0.18 \mathrm{~A}$

74

$11.3 \pm 2.3 \mathrm{c}$ 6.4-18.0

$0.20 \mathrm{~A}$

98 
Elytron length (mm)

$\begin{array}{ll}\text { Mean } \pm \mathrm{SD}^{3} & 4.19 \\ \text { Range } & 3.55 \\ C V^{4} & 0.06 \\ n & 146 \\ \text { Elytra width (mm) } & \\ \text { Mean } \pm \mathrm{SD}^{7} & 2.37 \\ \text { Range } & 1.96 \\ C V^{4} & 0.06 \\ n & 146 \\ \text { Pronotum length (mm) } \\ \text { Mean } \pm \mathrm{SD}^{7} \\ \text { Range } \\ C V^{4} \\ n \\ \text { Pronotum width (mm) } \\ \text { Mean } \pm \mathrm{SD}^{7} \\ \text { Range } \\ C V^{4} \\ n \\ \text { Head capsule width (mm) } \\ \text { Mean } \pm \mathrm{SD}^{7} \\ \text { Range } \\ C V^{4} \\ n \\ \text { Hind tibia length (mm) } \\ \text { Mean } \pm \mathrm{SD}^{7} \\ \text { Range } \\ C V^{4} \\ n\end{array}$

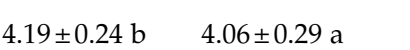

(55-4.65

$4.06 \pm 0.29$
$3.12-4.65$

$3.12-4.65$
$0.07 \mathrm{~A}$

150

$37 \pm 0.15$ a $\quad 2.38 \pm 0.14 a$

(1.75

$.06 \mathrm{~A}$

$2.38 \pm 0.14$
$2.02-2.75$

$2.02-2.75$
$0.06 \mathrm{~A}$

150

$1.15 \pm 0.07 \mathrm{a}$

$0.98-1.29$

$0.06 \mathrm{~A}$

150

$1.43 \pm 0.09 \mathrm{a}$

$1.16-1.65$

$0.07 \mathrm{~A}$

150

$1.15 \pm 0.06 \mathrm{a} \quad 1.24 \pm 0.07 \mathrm{~b}$

$0.98-1.29 \quad 1.04-1.41$

$0.05 \mathrm{~A}$

150

$1.73 \pm 0.11 \mathrm{a} \quad 1.81 \pm 0.12 \mathrm{~b}$

$1.30-1.90$

$0.06 \mathrm{~A}$

150
$0.05 \mathrm{~A}$

196

$1.40-2.10$

$4.32 \pm 0.28 \mathrm{c}$
$3.43-5.08$
$0.07 \mathrm{~A}$
196

$2.53 \pm 0.16 \mathrm{~b}$
$1.96-2.94$
$0.06 \mathrm{~A}$
196

$1.21 \pm 0.07 \mathrm{a}$
$0.98-1.35$
$0.06 \mathrm{~A}$
196

$1.51 \pm 0.10 \mathrm{~b}$
$1.22-1.71$
$0.06 \mathrm{~A}$
196
$1.24 \pm 0.07 \mathrm{~b}$
$1.04-1.41$
$0.05 \mathrm{~A}$
196
$1.81 \pm 0.12 \mathrm{~b}$
$1.40-2.10$
$0.07 \mathrm{~A}$
196

196

$\begin{array}{lll}4.32 \pm 0.20 \mathrm{a} & 4.16 \pm 0.28 \mathrm{a} & 4.39 \pm 0.28 \mathrm{a} \\ 3.86-4.65 & 3.43-4.65 & 3.73-5.08 \\ 0.05 \mathrm{~A} & 0.07 \mathrm{~B} & 0.06 \mathrm{AB} \\ 73 & 75 & 98 \\ & & \\ 2.44 \pm 0.14 \mathrm{a} & 2.42 \pm 0.15 \mathrm{a} & 2.56 \pm 0.15 \mathrm{~b} \\ 2.20-2.75 & 2.02-2.75 & 2.02-2.94 \\ 0.06 \mathrm{~A} & 0.06 \mathrm{~A} & 0.06 \mathrm{~A} \\ 73 & 75 & 98 \\ & & \\ & 1.17 \pm 0.06 \mathrm{a} & 1.22 \pm 0.06 \mathrm{a} \\ & 0.98-1.29 & 1.04-1.35 \\ & 0.05 \mathrm{~A} & 0.05 \mathrm{~A} \\ & 75 & 98 \\ & & \\ & 1.47 \pm 0.08 \mathrm{a} & 1.54 \pm 0.10 \mathrm{~b} \\ & 1.22-1.65 & 1.29-1.71 \\ & 0.06 \mathrm{~A} & 0.06 \mathrm{~A} \\ & 75 & 98 \\ & & \\ & 1.17 \pm 0.06 \mathrm{a} & 1.24 \pm 0.07 \mathrm{~b} \\ & 0.98-1.29 & 1.04-1.41 \\ & 0.05 \mathrm{~A} & 0.06 \mathrm{~A} \\ & 75 & 98 \\ & & \\ & 1.74 \pm 0.10 \mathrm{a} & 1.82 \pm 0.12 \mathrm{~b} \\ 1.40-1.90 & 1.40-2.00 \\ & 0.06 \mathrm{~A} & 0.07 \mathrm{~A} \\ & 75 & 98\end{array}$

$4.05 \pm 0.20 \mathrm{~b}$
$3.55-4.53$
$0.05 \mathrm{~A}$

$0.05 \mathrm{~A}$

73

$2.30+0.12 a$

$1.96-2.51$

$0.05 \mathrm{~A}$

73

$\begin{array}{ll}3.95 \pm 0.25 \mathrm{a} & 4.25 \pm 0.27 \mathrm{c} \\ 3.12-4.41 & 3.43-4.77 \\ 0.06 \mathrm{~A} & 0.06 \mathrm{~A} \\ 75 & 98 \\ & \\ 2.35 \pm 0.13 \mathrm{a} & 2.49 \pm 0.16 \mathrm{~b} \\ 2.02-2.63 & 1.96-2.88 \\ 0.06 \mathrm{~A} & 0.06 \mathrm{~A} \\ 75 & 98 \\ & \\ 1.13 \pm 0.07 \mathrm{a} & 1.20 \pm 0.08 \mathrm{a} \\ 0.98-1.22 & 0.98-1.35 \\ 0.06 \mathrm{~A} & 0.06 \mathrm{~A} \\ 75 & 98 \\ & \\ 1.38 \pm 0.08 \mathrm{a} & 1.49 \pm 0.09 \mathrm{~b} \\ 1.16-1.59 & 1.22-1.65 \\ 0.06 \mathrm{~A} & 0.06 \mathrm{~A} \\ 75 & 98 \\ & \\ 1.14 \pm 0.06 \mathrm{a} & 1.24 \pm 0.06 \mathrm{~b} \\ 1.04-1.29 & 1.10-1.35 \\ 0.06 \mathrm{~A} & 0.05 \mathrm{~A} \\ 75 & 98 \\ 1.72 \pm 0.11 \mathrm{a} & 1.81 \pm 0.12 \mathrm{~b} \\ 1.30-1.90 & 1.50-2.10 \\ 0.06 \mathrm{~A} & 0.07 \mathrm{~A} \\ 75 & 98\end{array}$

75

Overall phenotypic $C V$

Mean \pm SD $^{8}$ $0.49 \pm 0.55 \mathrm{~A}$

$0.39 \pm 0.42 \mathrm{~A}$

$0.37 \pm 0.41 \mathrm{~A}$

$0.46 \pm 0.51^{9}$

$0.38 \pm 0.41^{9}$

$0.38 \pm 0.47^{9}$

$0.48 \pm 0.74 \mathrm{~A}$

$0.28 \pm 0.41 \mathrm{~A}$

$0.30 \pm 0.40 \mathrm{~A}$

${ }^{1} \mathrm{fdr}$-corrected multiple pairwise contrast comparisons with sequential Sidak procedure after GZLM for non-normally distributed data. Distribution considered as Poisson loglinear link function.

${ }^{2}$ Difference in variation through comparing variances using Levine's tests in case of non-normally distributed data.

${ }^{3}$ fdr-corrected multiple Games-Howell pairwise comparison post hoc test after ANCOVA because of close-to-normally distributed data (with or without transformation) and unequal homogeneity of variances.

${ }^{4}$ Difference in variation through comparing variances using F - statistics in case of normally distributed data (with or without transformation).

${ }^{5}$ fdr-corrected multiple pairwise contrast comparisons with sequential Sidak procedure after GZLM because of non-normally distributed data. Distribution considered as binary probit link function.

Differences in variances not tested because of lack of enough spread/level pairs.

7 fdr-corrected multiple comparison Tukey post hoc range test after ANCOVA because of close-to-normally distributed data (with or without transformation) and equal homogeneity of variances.

8 Differences of overall $\mathrm{CV}$ according to non-parametric related samples sign test.

9 Differences according to related samples Friedman's two-way ANOVA by ranks. Here significant effect $P=0.018$. 


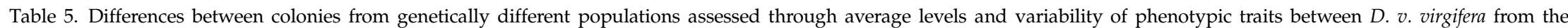
Central/Southeastern European genetic population (CSE, $\left.F_{2}\right)$, the Northwestern Italian genetic population (NW I, $\left.F_{2}\right)$, and the USA genetic population $\left(U S A, F_{8}\right)$. Different letters in rows indicate significant differences of mean values (small fonts) or variances (capital fonts) of traits between colonies according to $\mathrm{fdr}$ correction; $n=$ number of individuals, $\mathrm{SD}=$ standard deviation, $C V=$ coefficient of variation.

\begin{tabular}{|c|c|c|c|c|c|c|c|c|c|}
\hline \multirow[t]{2}{*}{ Phenotypic trait } & \multicolumn{3}{|l|}{ Both Sexes } & \multicolumn{3}{|l|}{ Females } & \multicolumn{3}{|l|}{ Males } \\
\hline & $\begin{array}{l}\text { NW I } \\
F_{2}\end{array}$ & $\begin{array}{l}\mathrm{CSE} \\
\mathrm{F}_{2}\end{array}$ & $\begin{array}{l}\text { USA } \\
F_{8}\end{array}$ & $\begin{array}{c}\mathrm{NW} \\
F_{2}\end{array}$ & $\begin{array}{c}\text { CSE } \\
F_{2}\end{array}$ & $\begin{array}{c}\text { USA } \\
F_{8}\end{array}$ & $\begin{array}{c}\text { NW I } \\
F_{2}\end{array}$ & $\begin{array}{c}\text { CSE } \\
F_{2}\end{array}$ & $\begin{array}{c}\text { USA } \\
F_{8}\end{array}$ \\
\hline \multicolumn{10}{|c|}{ Fitness traits } \\
\hline Mean $\pm S^{1}$ & & & & $747 \pm 442 \mathrm{a}$ & $579 \pm 361 \mathrm{a}$ & $691 \pm 396$ a & & & \\
\hline Range & & & & $0-1323$ & $0-1507$ & $0-1489$ & & & \\
\hline $\mathrm{CV}^{2}$ & & & & $0.59 \mathrm{~A}$ & $0.62 \mathrm{~A}$ & $0.57 \mathrm{~A}$ & & & \\
\hline$n$ & & & & 19 & 74 & 73 & & & \\
\hline \multicolumn{10}{|c|}{ Egg overwintering survival (\%) } \\
\hline Mean $\pm \mathrm{SD}^{3}$ & $65 \pm 11 \mathrm{c}$ & $42 \pm 17$ a & $43 \pm 27 b$ & & & & & & \\
\hline Range & $51-90$ & $4-82$ & $0-100$ & & & & & & \\
\hline $\mathrm{CV}^{2}$ & $0.17 \mathrm{~A}$ & $0.41 \mathrm{AB}$ & $0.63 \mathrm{~B}$ & & & & & & \\
\hline$n$ clutches & 16 & 70 & 71 & & & & & & \\
\hline \multicolumn{10}{|c|}{ Hatched-larva-to-adult survival (\%) } \\
\hline Mean $\pm \mathrm{SD}^{4}$ & $10 \pm 0$ & $18 \pm 12$ & $21 \pm 3$ & & & & & & \\
\hline Range & $10-10$ & $6-29$ & $19-24$ & & & & & & \\
\hline $\mathrm{CV}^{5^{\circ}}$ & 0.00 & 0.67 & 0.13 & & & & & & \\
\hline$n$ clutches & 16 & 70 & 72 & & & & & & \\
\hline \multirow{2}{*}{\multicolumn{10}{|c|}{ Egg-to-adult survival (\%) }} \\
\hline Mean $\pm \mathrm{SD}^{3}$ & $7 \pm 1 \mathrm{a}$ & $7 \pm 6$ a & $9 \pm 5 \mathrm{a}$ & & & & & & \\
\hline Range & $5-9$ & $0-19$ & $0-19$ & & & & & & \\
\hline $\mathrm{CV}^{5^{\circ}}$ & 0.17 & 0.79 & 0.56 & & & & & & \\
\hline$n$ clutches & 16 & 70 & 71 & & & & & & \\
\hline \multicolumn{10}{|c|}{ Adult lifespan (days) (standardized to 70 days) } \\
\hline Mean $\pm \mathrm{SD}^{1}$ & $62 \pm 18$ a & $61 \pm 18 \mathrm{a}$ & $59 \pm 18$ a & $68 \pm 5$ a & $59 \pm 18$ a & $58 \pm 16$ a & $57 \pm 24$ a & $62 \pm 18$ a & $60 \pm 19 a$ \\
\hline Range & $3-70$ & $4-70$ & $3-70$ & $55-70$ & $4-70$ & & & & $3-70$ \\
\hline$C V^{2}$ & $0.29 \mathrm{~A}$ & $0.29 \mathrm{~A}$ & $0.30 \mathrm{~A}$ & $0.07 \mathrm{~A}$ & $0.30 \mathrm{~B}$ & $0.28 \mathrm{~B}$ & $0.43 \mathrm{~B}$ & $0.28 \mathrm{~A}$ & $0.32 \mathrm{~A}$ \\
\hline$n$ & 38 & 148 & 146 & 19 & 74 & 73 & 19 & 74 & 73 \\
\hline \multirow{2}{*}{\multicolumn{10}{|c|}{$\begin{array}{l}\text { Initial activity trait } \\
\text { Proportion of adults flving (\%) }\end{array}$}} \\
\hline \multicolumn{6}{|c|}{ Proportion of adults flying (\%) } & & & & \\
\hline Mean $\pm \mathrm{SD}^{6}$ & $94 \pm 23 \mathrm{a}$ & $90 \pm 31$ a & $82 \pm 39 a$ & $89 \pm 32 \mathrm{a}$ & $85 \pm 36 \mathrm{a}$ & $71 \pm 46$ a & $100 \pm 0 \mathrm{a}$ & $94 \pm 23 \mathrm{a}$ & $93 \pm 26$ a \\
\hline$C V^{2}$ & $0.25 \mathrm{~A}$ & $0.34 \mathrm{AB}$ & $0.48 \mathrm{~B}$ & $0.35 \mathrm{~A}$ & $0.42 \mathrm{AB}$ & $0.65 \mathrm{~B}$ & $0.0 \mathrm{~A}$ & $0.24 \mathrm{~B}$ & $0.28 \mathrm{~B}$ \\
\hline$n$ & 36 & 145 & 146 & 19 & 73 & 72 & 17 & 72 & 69 \\
\hline \multicolumn{10}{|c|}{ Flight take - off response (seconds) } \\
\hline Mean $\pm \mathrm{SD}^{1}$ & $17 \pm 26 \mathrm{a}$ & $41 \pm 61 \mathrm{~b}$ & $49 \pm 79 \mathrm{~b}$ & $28 \pm 31 \mathrm{a}$ & $51 \pm 62 \mathrm{a}$ & $51 \pm 77 \mathrm{a}$ & $5 \pm 7 \mathrm{a}$ & $30 \pm 59 a$ & $46 \pm 82 \mathrm{a}$ \\
\hline Range & $1-112$ & $1-290$ & $1-299$ & $2-112$ & $1-263$ & $1-297$ & $1-28$ & $1-290$ & $1-299$ \\
\hline$C V^{2}$ & $1.50 \mathrm{~A}$ & $1.49 \mathrm{~B}$ & $1.63 \mathrm{C}$ & $1.11 \mathrm{~A}$ & $1.20 \mathrm{~B}$ & $1.50 \mathrm{C}$ & $1.40 \mathrm{~A}$ & $1.96 \mathrm{~B}$ & $1.79 \mathrm{~B}$ \\
\hline n & 36 & 144 & 139 & 19 & 73 & 71 & 17 & 71 & 68 \\
\hline \multirow{2}{*}{\multicolumn{10}{|c|}{ Initial morphometric traits }} \\
\hline & & & & & & & & & \\
\hline Mean $\pm \mathrm{SD}^{7}$ & $9.0 \pm 2.0 \mathrm{a}$ & $11.7 \pm 1.9 \mathrm{c}$ & $10.9 \pm 2.0 \mathrm{~b}$ & $9.9 \pm 2.0 \mathrm{a}$ & $12.3 \pm 1.6 \mathrm{~b}$ & $11.6 \pm 2.0 \mathrm{a}$ & $8.2 \pm 1.6 \mathrm{a}$ & $11.0 \pm 1.9 \mathrm{c}$ & $10.1 \pm 1.8 \mathrm{~b}$ \\
\hline Range & $4.5-13.0$ & $4.9-18.2$ & $6.3-20.0$ & $6.2-13.0$ & $9.5-18.2$ & $8.0-20.0$ & $4.5-10.7$ & $4.9-15.6$ & $6.3-15.5$ \\
\hline$C V^{8}$ & $0.22 \mathrm{~A}$ & $0.16 \mathrm{~A}$ & $0.19 \mathrm{~A}$ & $0.20 \mathrm{~A}$ & $0.13 \mathrm{~A}$ & $0.17 \mathrm{~A}$ & $0.19 \mathrm{~A}$ & $0.17 \mathrm{~A}$ & $0.18 \mathrm{~A}$ \\
\hline n & 38 & 148 & 146 & 19 & 74 & 73 & 19 & 74 & 73 \\
\hline \multicolumn{10}{|c|}{ Elytron length (mm) } \\
\hline Mean $\pm \mathrm{SD}^{8}$ & & & & $4.32 \pm 0.32 \mathrm{a}$ & $4.45 \pm 0.15 \mathrm{a}$ & $4.32 \pm 0.20 \mathrm{a}$ & $4.11 \pm 0.22 \mathrm{a}$ & $4.26 \pm 0.22 \mathrm{~b}$ & $4.05 \pm 0.20 \mathrm{a}$ \\
\hline Range & $3.67-4.84$ & $3.67-4.90$ & $3.55-4.65$ & $3.79-4.84$ & $4.10-4.90$ & $3.86-4.65$ & $3.67-4.41$ & $3.67-4.71$ & $3.55-4.53$ \\
\hline$C V^{8}$ & $0.07 \mathrm{~B}$ & $0.05 \mathrm{~A}$ & $0.06 \mathrm{AB}$ & $0.08 \mathrm{~B}$ & $0.03 \mathrm{~A}$ & $0.05 \mathrm{AB}$ & $0.05 \mathrm{~A}$ & $0.05 \mathrm{~A}$ & $0.05 \mathrm{~A}$ \\
\hline$n$ & 38 & 148 & 146 & 19 & 74 & 73 & 19 & 74 & 73 \\
\hline
\end{tabular}


until take-off (for those that flew) were calculated for young adult males and females by colony.

\section{Initial morphometric traits}

In total, seven commonly used morphometric traits were measured on each individual D. $v$. virgifera within $24 \mathrm{~h}$ following adult emergence (=initial morphometric traits without feeding), because measures on young adults more easily allow the detection of differences between individuals or colonies than measures on mature adults which are more variable (Li et al., 2009, 2010). The tested young adults were assumed to no longer be teneral, as they were fully coloured and did not have the light grey and soft body typical of newly emerged adults.

Fresh body weight was measured by transferring adults into a small plastic container (h:50 mm, dia.: $27 \mathrm{~mm}$ ) and weighing them on a $0.1 \mathrm{mg}-160 \mathrm{~g}$ precision scale (Fox \& Czesak, 2006) and then returning them to the rearing container.

Other measured traits included: head capsule width including eyes (Branson \& Ortman, 1970; Branson \& Sutter, 1985), pronotum width and length, elytra width (i.e., single measurement of both elytra together across the dorsum), elytron length (randomly either left or right, Mabry et al., 2004), as well as hind tibia length (randomly either left or right, Jenner \& Kuhlmann, 2006). Adults were placed on a cool pad (cool but not frozen Icepack, Migros, Delemont, Switzerland) to limit their activity during the measurements with a micrometer scale to the nearest $0.06 \mathrm{~mm}$ under a stereomicroscope (16× magnification) ( $\mathrm{Li}$ et al., 2010). The individuals were returned to the bioassay containers.

\section{Comparing averages of phenotypic traits}

Averages of the fitness, activity, and morphometric trait levels were calculated for each colony and, per female, per male and for pooled male and female data. Distributions of these data were investigated using histograms as well as normal and detrended normal probability $Q-Q$ plots (Kinnear \& Gray, 2000).

Data on fresh body weight, elytra length and width, head capsule width, pronotum length and width, and hind tibia length had normal distributions. Data on elytron length and elytra width were standardized ((Trait data-Mean of trait data)/ Variance of trait data).

Data on overwintering survival of eggs as well as eggto-adult survival were normally distributed after $\operatorname{sqrt}(x+1)$ transformation.

Data on adult lifespan, fecundity, flight take-off response, and crawling speed were Poisson distributed. Proportion of adults flying and crawling followed a binomial distribution. Larval-to-adult survival had too few data points to check for normality, and to allow parametric tests.

The influence of the independent explanatory factors 'colony origin', 'genetic population', 'generation', 'sex' and their interactions were tested on each trait (dependent factor). For normally distributed trait data, ANCOVA (Univariate general linear modelling) was applied. Since trait correlations can seriously confound factorial analyses of individual traits (Lande \& Arnold, 1983), associations between phenotypic traits, i.e., the bivariate correlations published by Li et al. (2009, 2010), were taken as covariates in the analyses (i.e., the fresh body weight with pronotum length, elytron length and elytra width, and vice versa; pronotum length with pronotum width 
and vice versa; the egg overwintering survival with fecundity).

For non-normally distributed trait data, generalized linear models (GZLM) were applied taking the distribution type of trait data into account (e.g., adult lifespan: Poisson distribution with a log linear link function; fecundity: Poisson, log linear; proportion of adults flying and crawling: binary, probit; flight take-off and crawling speed: both Poisson, log linear). During this procedure, correlated traits were considered as covariates (e.g., adult lifespan with fecundity; flight take-off response with pronotum length; crawling speed with body weight, hind tibia length, and pronotum width).

Once a significant factorial effect was detected by the above described models, the averages of the phenotypic trait levels were compared between colonies. For this, Tukey post hoc range test was used in the case of normally distributed trait data and equal homogeneity of variances (Kinnear \& Gray, 2000); the Games-Howell pairwise comparison post hoc test in the case of normally distributed trait data with unequal homogeneity of variances (Games \& Howell, 1976); or multiple pairwise contrast comparisons with a sequential Sidak procedure (in GZLM) in the case of non-normally distributed trait data. Due to the large number of compared factors, the $P$-values obtained from the posthoc tests were adjusted to $q$-values to reduce the probability of Type I errors of $P$ values using the Benjamini-Hochberg method (Benjamini \& Hochberg, 1995) through the package Q-VALUE (Storey, 2002; Storey \& Tibshirani, 2003) in R (R Development Core Team, 2009).

\section{Comparing variability in phenotypic traits}

Standard deviations and variances of data were computed for the fitness, activity, and morphometric traits for each colony and per female, per male and for pooled male and female data (Valladares et al., 2006). Distributions of trait data were investigated using histograms as well as normal and detrended normal probability $Q-Q$ plots (Kinnear \& Gray, 2000).

Variability of each trait was compared with $F$ - statistics for homogeneity of variances (based on means) in the case of normally distributed data, and Levine's tests for homogeneity of variances in the case of non-normally distributed data. Due to the large number of compared traits, a false discovery rate $(\mathrm{fdr})$ analyses was applied to reduce the number of false $P$ values $<0.05$ from the variance tests (see above).

To understand the standard deviation in the context of a mean of data, the coefficient of variation $(\mathrm{CV})$ was calculated as follows: $C V=$ Standard deviation of trait/Mean of trait (Krebs, 1994). As the $C V$ is a dimensionless number (independent from units or widely different mean values), the $C V$ of tested traits could be averaged per colony to characterize the overall phenotypic variability. This overall phenotypic variability was compared between colonies using related samples Wilcoxon Signed Rank test.

\section{Results}

\section{Phenotypic traits}

\section{Fitness traits}

D. $v$. virgifera females laid $565 \pm 174($ mean \pm SD) eggs on average across all colonies under standardized laboratory conditions within 70 days of age (tables 2-5). At 70 days, the maximum individual fecundity was 1507 eggs (found in a CSE $3 F_{2}$ colony). At 98 days, the maximum was 1814 eggs (found in a USA $F_{191}$ colony) which may be considered as the approximate maximum potential fecundity of this species. A small proportion of females in each colony did not lay any eggs.

Across all colonies, $38 \pm 17 \%$ of eggs successfully overwintered under standardized laboratory conditions, the range for clutches was from 0 to $100 \%$. Larva-to-adult survival was $19 \pm 9 \%$ with a maximum of $39 \%$ and a minimum of $6 \%$. Eggto-adult survival was $7 \pm 1 \%$ with a maximum of $19 \%$ and a minimum of $0 \%$. Since adult lifespan was standardized to 70 days, no data on maximum lifespan were obtained. Average standardized lifespan was $56 \pm 7$ days across colonies (females $57.6 \pm 6.5$ days, males $54.8 \pm 8.0$ days).

The average generational growth rate (i.e., the net reproductive rate) was $27 \pm 2$ times across colonies under standardized laboratory conditions, with a maximum rate of 100 for a few D. v. virgifera pairs (found in a CSE $F_{2}$ colony and in a USA $F_{8}$ colony) and a minimum rate of 0 for a few other pairs.

\section{Initial activity traits}

On average across colonies, $99 \pm 1 \%$, of the tested young (i.e., 7-day-old) adults crawled, and on average escaped the $400-\mathrm{mm}$ long tube within $30 \pm 5 \mathrm{~s}$ (speed $=13 \mathrm{~mm} \mathrm{~s}^{-1}$ or $8 \mathrm{~m} \mathrm{~min}^{-1}$ ). Among females, $98 \pm 2 \%$ crawled and escaped the tube within $32 \pm 6 \mathrm{~s}$. Among males, $99 \pm 1 \%$ crawled and escaped the tube this within $29 \pm 6 \mathrm{~s}$. The fastest adults needed only about $9-10 \mathrm{~s}$ to travel the $400 \mathrm{~mm}$ tube distance (speed $=40-44 \mathrm{~mm} \mathrm{~s}^{-1}$ or about $25 \mathrm{~m} \mathrm{~min}^{-1}$ ). Such individuals were found among both sexes and in each colony.

On average across colonies, $83 \pm 17 \%$ of the young adults flew off the flight stands under standardized laboratory conditions. The mean time between release and take-off was $44 \pm 24$ s. Among females, $75 \pm 22 \%$ took off, on average this occurred $54 \pm 21 \mathrm{~s}$ after they were released on the stand. Males flew off more frequently than females $(91 \pm 10 \%$, $t$-test $=9$, df $1 ; 997, P=0.003$ ) and faster (within on average $33 \pm 27 \mathrm{~s}$, $\left.t=-10.5, \mathrm{df}_{1 ; 890}, P=0.004\right)$. The very fastest adults needed only ca. $1 \mathrm{~s}$ to take off, and such individuals were found among both sexes and in each colony.

\section{Initial morphometric traits}

Young, i.e., 1-day-old adults weighed $10.4 \pm 1.1 \mathrm{mg}$ on average across colonies under standardized laboratory conditions. Young females were heavier $(11.01 \pm 0.9 \mathrm{mg})$ than young males $(9.8 \pm 1.1 \mathrm{mg}$, $t$-test, $P=0.007)$. The heaviest young female was $20 \mathrm{mg}$ (found in a USA $F_{8}$ colony) and the heaviest young male was weighed $18 \mathrm{mg}$ (found in a USA $F_{191}$ colony). The lightest young female was $6.2 \mathrm{mg}$ (found in a NW I $F_{2}$ colony), the lightest young male was $3.4 \mathrm{mg}$ (found in a USA $F_{23}$ colony).

One-day-old adults had $4.2 \pm 1.1 \mathrm{~mm}$ long and $2.43 \pm$ $0.13 \mathrm{~mm}$ wide elytra, a $1.2 \pm 0.03 \mathrm{~mm}$ long and $1.48 \pm 0.05 \mathrm{~mm}$ wide pronotum, a $1.19 \pm 0.03 \mathrm{~mm}$ wide head capsule, and $1.77 \pm 0.04 \mathrm{~mm}$ long hind tibias. For separate average measures by sexes refer to tables $2-5$. The longest female elytron was $5.1 \mathrm{~mm}$, and the longest male elytron was $4.77 \mathrm{~mm}$ (both from the USA $F_{191}$ colony). The widest female elytra were $2.94 \mathrm{~mm}$ and the widest male elytra were $2.88 \mathrm{~mm}$ (both from the USA $F_{191}$ colony). The shortest female elytron was $3.43 \mathrm{~mm}$ 
(from the USA $F_{23}$ colony), and the shortest male elytron was $2.45 \mathrm{~mm}$ (from the NW I $F_{1}$ colony). The narrowest female elytra were $1.96 \mathrm{~mm}$, and the narrowest male elytra were $1.90 \mathrm{~mm}$ (both were from the NW I $F_{1}$ colony).

\section{Differences between $\mathrm{F}_{1}$ colonies from different locations of the same population: field-influenced parental effects}

The different natal fields of $F_{0} D . v$. virgifera from the same genetic population (i.e., CSE European population) affected the average of phenotypic trait levels in the subsequently laboratory-reared $F_{1}$ colonies for 7 out of 15 traits (47\%) (tables 2-6). This field-influenced parental effect was mainly apparent for fitness traits (e.g., 70- and 98-day fecundity, adult life span), and to some extent for morphometric traits (elytron length, head capsule width, and hind tibia length) (table 2). The only activity trait that differed between $F_{1}$ colonies was their proportion of adults flying $(14 \%$ difference between the two tested colonies, table 2).

The environmental conditions in different natal fields of a colony from the same genetic population (i.e., CSE) affected the overall phenotypic variability of the subsequently laboratory-reared $F_{1}$ (i.e., overall $C V$ of 0.38 for colony CSE1 versus 0.49 for colony CSE2, related samples sign test $P=0.006$, table 2). The variability of more than half of the examined traits (10 out of $15 ; 67 \%$ ) was affected by the original environmental conditions of the natal field (tables 2 and 6). This effect was apparent for most morphometric traits (adult fresh body weight, elytron length, pronotum length and width, and hind tibia length) as well as fitness traits (70- and 98-day fecundity, egg overwintering survival, and adult life span) (table 2). Except for the proportion of adults flying, natal fields had little effect on the variability of activity traits.

\section{Differences between the $\mathrm{F}_{1}$ and $\mathrm{F}_{2}$ generation of the same population: effects of early generation laboratory rearing}

$F_{2}$ generation D. v. virgifera from the NW Italian population differed from the corresponding $F_{1}$ of the same colony in the average levels of 5 out of $9(56 \%)$ of the phenotypic traits (tables 3 and 6). Effects of such early generation laboratory rearing were mainly apparent for morphometric traits, (i.e., the $F_{2}$ adults were usually slightly lighter, but with slightly longer and wider elytra than their $F_{1}$ ). Effects were also apparent for fitness traits (i.e., the $F_{2}$ adults usually laid more eggs, and their eggs overwintered more successfully than the $\left.F_{1}\right)$. No such effects were apparent among activity traits.

Early generation laboratory rearing did not affect the overall phenotypic variability of the colony (i.e., overall $\mathrm{CV}$ of 0.52 of $F_{1}$ versus 0.42 of $F_{2}$, related samples sign test $P=0.453$, table 3). The variability of only three out of eight phenotypic traits (38\%) was significantly affected (tables 3 and 6). For example, $F_{2}$ and $F_{1}$ adults had similar variability in their fecundity and adult life span, but the $F_{2}$ were less variable in their egg overwintering survival than the $F_{1}$ (table 3 ). $F_{2}$ and $F_{1}$ adults also had similar variability in their flight takeoff response, but the $F_{2}$ adults were more variable in the proportion of adults flying than the $F_{1} . F_{2}$ and $F_{1}$ adults had similar variability in their elytra lengths and widths, but the $F_{2}$ adults were more variable in their fresh body weight than the $F_{1}$.
Differences between different generations of the same population: effects of long-term laboratory rearing

The generation number of laboratory reared $D$. $v$. virgifera colonies (i.e., $F_{8}, F_{23}$, and $F_{191}$ USA populations) affected the average levels of 9 out of $15(60 \%)$ measured phenotypic traits (tables 4 and 6). Long-term rearing mainly affected morphometrics. For example, $F_{191}$ adults generally appeared larger and heavier than the $F_{8}$ and $F_{23}$ adults (i.e., fresh body weight, elytra length and width, pronotum width, head capsule width, and hind tibia length, table 4). Some differences between $F_{8}$ and $F_{23}$ were also detected, but seem not to depend on generation numbers. Long-term rearing also significantly affected some fitness and activity traits. For example, $F_{191}$ adults generally appeared to have a greater 98-day fecundity than the $F_{23}$ adults $(703 \pm 488$, range $0-1814$, versus $537 \pm 390$, range $0-1438$ ). But $F_{191}$ adults were less likely to fly and were slower to initiate flight than the $F_{23}$ and $F_{8}$ adults.

In contrast to effects on average levels of traits, long-term rearing usually did not affect the variability of traits. Only 3 out of $14(21 \%)$ traits had differences in overall phenotypic $C V$ s (Friedman's two-way ANOVA by ranks $P>0.05$, tables 4 and 6). Moreover, the slight increase in variability in the proportion of adults flying with increasing generation number is a result of fewer adults flying among longer-reared D. v. virgifera colonies.

\section{Differences between colonies from genetically different populations}

The three populations with different origins (i.e., NW Italian, CSE European, and USA) differed in the average levels of five out of nine (55\%) of the phenotypic traits (tables 5 and 6). The population origin mainly affected the morphometrics. For example, the CSE European adults were usually slightly heavier and larger than the adults of the other populations (table 5). Population origin had little effect on fitness and activity traits. For example, the NW Italian eggs seemed to more successfully overwinter than the USA eggs, and both overwintered more successfully than eggs of the CSE European population. Moreover, the NW Italian adults took off slightly faster than other adults, there was no take-off difference between CSE European and USA adults.

The three genetic populations differed in the variability of five out of eight traits (63\%) (tables 5 and 6). This was particularly true for activity traits, to some extent for morphometric traits, but less obvious for fitness traits (table 5). Considering that the USA population was the genetically most variable among the three tested populations, it had surprisingly low phenotypic variability. The USA population only appeared more variable in their egg overwintering survival and activity traits than the European populations. Consequently, no significant effect of a populations' original genetics was detected on the overall phenotypic variability of their laboratory colonies (i.e., differences in overall phenotypic $C V$ s; Friedman's two-way ANOVA by ranks $P>0.05$, table 5).

\section{Discussion}

There is a debate among researchers as to whether laboratory bioassays and comparative behavioural studies should be conducted with insects directly collected from the field or with insects reared over several generations under standardized laboratory conditions (Richerson \& Cameron, 
Table 6. Summary of effects of the original natal field, laboratory rearing, and the neutral genetic characteristics of the populations at the origin on averages (Av.) and variability (Var.) of phenotypic traits of young immature adults of Diabrotica $v$. virgifera based on significant differences shown in tables 2 to $5:+=$ significant effect at $P<0.05,++=$ significant effect at $P<0.005,0=$ no significant difference, empty = no data. With the exception of fecundity, only pooled data for both sexes is considered.

\begin{tabular}{|c|c|c|c|c|c|c|c|c|c|c|c|c|c|c|c|c|c|}
\hline \multirow[t]{2}{*}{ Effects } & & \multicolumn{5}{|c|}{ Fitness traits } & \multicolumn{4}{|c|}{ Initial activity traits } & \multicolumn{7}{|c|}{ Initial morphometric traits } \\
\hline & & 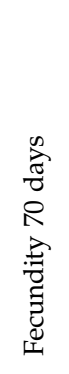 & 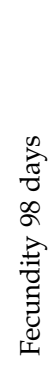 & 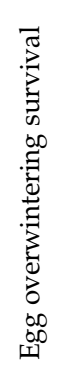 & 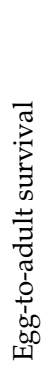 & 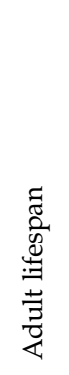 & 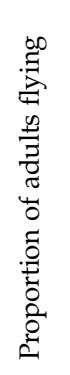 & 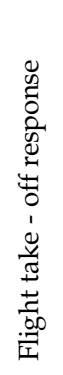 & 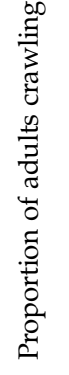 & 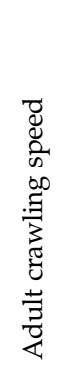 & 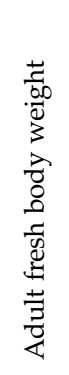 & 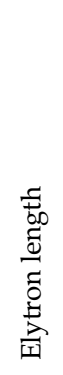 & 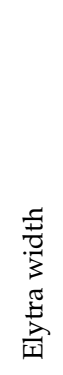 & 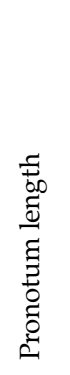 & 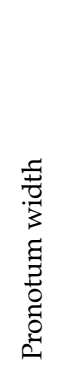 & 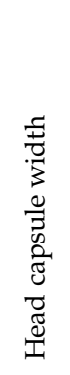 & 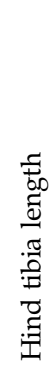 \\
\hline Field-influenced parental effect ${ }^{1}$ & Av. & + & + & 0 & & + & + & 0 & 0 & 0 & 0 & + & 0 & 0 & 0 & ++ & ++ \\
\hline Effects of early generation laboratory rearing ${ }^{2}$ & $\begin{array}{l}\text { Var. } \\
\text { Av. } \\
\text { Var. }\end{array}$ & $\begin{array}{l}++ \\
+ \\
0\end{array}$ & + & $\begin{array}{l}++ \\
++ \\
+\end{array}$ & 0 & $\begin{array}{l}++ \\
0 \\
0\end{array}$ & $\begin{array}{l}+ \\
0 \\
+\end{array}$ & $\begin{array}{l}0 \\
0 \\
0\end{array}$ & 0 & 0 & $\begin{array}{l}+ \\
++ \\
+\end{array}$ & $\begin{array}{l}+ \\
+ \\
0\end{array}$ & $\begin{array}{l}0 \\
++ \\
0\end{array}$ & + & ++ & 0 & + \\
\hline Effects of long-term laboratory rearing ${ }^{3}$ & $\begin{array}{l}\text { Av. } \\
\text { Var. }\end{array}$ & $\begin{array}{l}0 \\
0\end{array}$ & $\stackrel{+}{0}$ & $\begin{array}{l}0 \\
+\end{array}$ & & $\begin{array}{l}0 \\
0\end{array}$ & $\begin{array}{l}+ \\
+\end{array}$ & $\begin{array}{l}+ \\
+\end{array}$ & 0 & $\begin{array}{l}0 \\
0\end{array}$ & $\stackrel{++}{0}$ & + & $\stackrel{++}{0}$ & $\begin{array}{l}0 \\
0\end{array}$ & $\stackrel{++}{0}$ & $\stackrel{++}{0}$ & $\begin{array}{l}++ \\
0\end{array}$ \\
\hline Differences between colonies from genetically different populations ${ }^{4}$ & $\begin{array}{l}\text { Av. } \\
\text { Var. }\end{array}$ & $\begin{array}{l}0 \\
0\end{array}$ & & $\begin{array}{l}++ \\
+\end{array}$ & 0 & $\begin{array}{l}0 \\
0\end{array}$ & $\begin{array}{l}0 \\
+\end{array}$ & $\begin{array}{l}+ \\
++\end{array}$ & & & $\begin{array}{l}++ \\
0\end{array}$ & $\begin{array}{l}++ \\
+\end{array}$ & $\begin{array}{l}++ \\
+\end{array}$ & & & & \\
\hline
\end{tabular}

\footnotetext{
${ }^{1}$ Based on table 2

2 Based on table 3 .

3 Based on table 4

4 Based on table 5 .
} 
1974; Prokopy et al., 1975; Rossler, 1975; Bush et al., 1976; Huettel, 1976; Boiler \& Chambers, 1977; Chambers, 1977; Diamond et al., 2010). Our study shows that indeed nearly all of 16 tested phenotypic traits of seven different colonies of the maize pest $D$. $v$. virgifera can vary depending on the natal field of the collected specimens, on the genetic population as well as on short- and long-term laboratory rearing. The advantage of field collected insects might be that they had not adapted to certain laboratory conditions (Rossler, 1975). In fact, the variability inherent in field populations due to field conditions is why studies with pest insects from the field, such as $D$. $v$. virgifera, are so very relevant. If our intent is to understand the characteristics and vulnerabilities of the insects that plague our crops, the place to seek answers is among the pests in situ. However, possible differences originating from the natal environmental field conditions may hinder comparative studies (Diamond et al., 2010), something that our results show to be considered also for D. v. virgifera. As for D. v. virgifera, researchers could use and refer to a number of colonies reared in different laboratories (Lefko et al., 2008) or could easily collect specimens from maize fields. Therefore, we have compared a number of different phenotypic traits among seven different $D$. $v$. virgifera colonies. However, the colonies and the combinations we used for comparisons were not comprehensive. There are many more available colonies maintained at a relatively large number of existing rearing facilities (e.g., USDA-ARS NCARL, Brookings, South Dakota, USA; Biotest laboratories, Sagerheide, Germany; French Agricultural Research, Lamberton, MN, USA; CABI, Delemont, Switzerland and Hodmezovasarhely, Hungary; and others), and the options for field-collected $D$. v. virgifera populations are only limited by the number of heavily infested maize fields.

Many phenotypic traits are sensitive to conditions in the field environment. For example, fitness and activity of $D$. v. virgifera are affected by recent diet experience (Levine et al., 2002; Mabry et al., 2004). Differences in weather conditions such as temperature can increase or decrease developmental time and influence survival (Toepfer et al., 2005). Biological attributes such as insect age also affect phenotypic traits (Li et al., 2009). Therefore, in our study, all of the D. $v$. virgifera were tested at a similar age, and were provided with the same type and amount of food. They were reared under similar laboratory conditions based on George \& Ortman (1965) and Branson et al. (1975). Still, there remains the possibility that slight variation in the controlled laboratory environment or in the quality of food might have occurred from one experimental series to the next. However, due to the large samples size and well-controlled experimental conditions used, we hope that our results can provide good indications about constancies or possible changes in traits that may occur due to influences from field conditions or laboratory-rearing. Our study stands out among other comparisons of populations because it focuses on quantities that might be more tangible than the genes and alleles that are usually the metric for comparing populations.

\section{Differences between $\mathrm{F}_{1}$ colonies from different locations of the same population: field-influenced parental effects}

While insects from the field may have the advantage that they have not been selected for adaptations to a variety of laboratory conditions (e.g., Bactrocera spp.,. Miyatake \& Yamagishi, 1999), they may differ from each other in traits due to variation in local field conditions. Indeed, our study revealed that the original and natal collection site of $F_{0}-$ $D$. v. virgifera can affect the average levels and the variability of phenotypic traits in the subsequently laboratory-reared $F_{1} c a$. 47 and $67 \%$ of the time, respectively (=parental or transgenerational effects). As the tested insects for these research questions originated from the same genetic population (i.e., the CSE European population), a genetic population effect can be excluded. Environmentally driven parental effects were mainly apparent in fitness and morphometric traits. Effects on fecundity might need to be specifically considered when conducting studies related to $D$. v. virgifera fitness. In contrast, averages as well as the variability in activity traits, remained largely stable from $F_{0}$ to $F_{1}$. Flying and crawling were behavioural phenotypic traits that varied little in our assays; perhaps behaviour is generally less influenced by field origin than fitness or morphometric traits. Whether this is indeed true for specific agriculture-relevant behavioural traits such as host plant finding, mating, and oviposition behaviour remains to be tested.

Differences between the $\mathrm{F}_{1}$ and $\mathrm{F}_{2}$ generation of the same population: effects of early generation laboratory rearing

In general for comparative studies, we advise laboratory rearing of field-collected $D$. $v$. virgifera until at least the $F_{2}$ generation to avoid the varying field-influenced parental effects on the fitness and morphometrics occurring in the $F_{0}$ or $F_{1}$. Indeed, our results showed that such rearing can affect the average levels and variability of phenotypic traits in 56 and $38 \%$ of cases, respectively. This was particularly obvious in a change in morphometrics and in an increase in fecundity and egg survival from $F_{1}$ to $F_{2}$, but less important for other fitness traits, or for activity traits. Interestingly, Lefko et al. (2008) also reported an increased egg survival as well as generational survival in early generations of the laboratory rearing of D. v. virgifera (i.e., during rearing from $F_{0}$ to approximately $F_{4}$ or $\left.F_{5}\right)$. It is unclear why some morphological traits changed in our study from $F_{1}$ to $F_{2}$; however, an increase in fitness characteristics can be understood. For example, a greater fecundity can result from more optimal larvae rearing conditions and subsequent adults for the $F_{1}$ (laying $F_{2}$ eggs) than for the field grown larvae and adults of the $F_{0}$ generation (laying $F_{1}$ eggs). From other insects it is also known that laboratory-reared colonies might mature more rapidly or have an increased reproductive rate (Miyatake \& Yamagishi, 1999). Therefore, laboratory colonies of $D$. $v$. virgifera reared over different numbers of generations may be comparable under similar conditions, as in other insects (Rossler, 1975; Diamond et al., 2010).

\section{Differences between different generations of the same population: effects of long-term laboratory rearing}

Insects in laboratory for long periods may undergo further changes due to certain selections (Richerson \& Cameron, 1974; Prokopy et al., 1975; Bush et al., 1976; Huettel, 1976; Boiler \& Chambers, 1977; Chambers, 1977; Hill \& Caballero, 1992; Stearns, 1992; Miyatake \& Yamagishi, 1999; Scannapieco et al., 2009; Khazaeli \& Curtsinger, 2010; Spurgeon, 2012), which may also affect the results of studies and hinder comparability. Some insects are known to have experienced drastic changes in both phenotype and genetics, when reared under artificial laboratory conditions (Rossler, 1975). One would expect that 
mass rearing of $D . v$. virgifera would select for higher reproductive rates (Mohaghegh et al., 1999), an effect that was only confirmed in the present study through the higher 98-day fecundity in the $F_{191}$ generation versus an earlier generation (e.g., $F_{23}$ ) but not for 70-day fecundity - a standard often used for D. v. virgifera (Li et al., 2009, 2010; Toepfer et al., 2012). Lefko et al. (2008) also found no consistent pattern of increased fecundity or fertility when rearing different laboratory colonies of $D$. v. virgifera up to $F_{11}$. From other insects it is known that colony fitness can strongly change due to genetic laboratory adaptation, and some may become so differentiated that reproductive barriers arise between them and wild populations (Rossler, 1975). Extreme differentiation has not been reported for $D$. v. virgifera. Even the non-diapause colony can still be easily crossed with wild $D$. v. virgifera after decades in culture (Lefko et al., 2008; Oswald et al., 2011).

In contrast to the relatively minor impacts on fitness, longterm rearing clearly reduced $D$. v. virgifera activity, and slightly increased body size and weight. Both types of traits are correlated (activity negatively with weight, activity positively with pronotum size and other morphometric characters, Li et al., 2010). Therefore, it is not clear whether the selection was on activity traits or on body weight-size or on both. Certainly the typical cages used for adult $D$. v. virgifera mass rearing do not allow or reward much flight, as they are usually of less than $0.5 \mathrm{~m}^{3}$ size, and cages contain several hundreds of adults (George \& Ortman, 1965; Branson et al., 1975). Moreover, there is little need to fly or crawl as food and sexual partners are always close to colony individuals. As above, flying and crawling are behavioural phenotypic traits, which might suggest that other behavioural traits could also be influenced by long-term rearing. In fact, conditions of rearing present few opportunities for any penalty for poor choices by the insect. Care should be taken when individuals from longterm reared laboratory colonies are used for behavioural studies (Richerson \& Cameron, 1974; Prokopy et al., 1975; Bush et al., 1976; Huettel, 1976; Boiler \& Chambers, 1977; Chambers, 1977). This caution should be extended to analyses of host plant acceptability/recognition or pheromonial responses (Tingey, 1986; Masson et al., 1987), topics that remain somewhat un-investigated for $D . v$. virgifera.

In contrast to the changes in average levels of some traits reported herein, long-term rearing did little to reduce the variability of traits, particularly not to morphometric variability. In contrast, Lefko et al. (2008) reported a marginal decline in overall phenotypic variation among laboratoryreared $D$. v. virgifera colonies, but the patterns were often inconsistent. Phenotypic variability could be expected to decrease because (i) environmental variability decreases under constant laboratory conditions and (ii) because multiple bottlenecks and inbreeding during laboratory rearing over many generations reduces genetic variability. Such genetic alterations could include the loss of alleles, decreases in heterozygosity, and shifts in allele frequencies (Masson et al., 1987; Norris et al., 2001). Kim et al. (2007) investigated the genetic variability of the USDA-ARS NCARL non-diapause colony of D. $v$. virgifera (>190 generations), versus several diapause colonies ( $>22$, and 3rd-8th generations), and versus wild populations. Astonishingly, the genetic variability (allele richness, expected and/or observed heterozygosity) was found to be similar among most of the diapause laboratory colonies as well as wild populations (Kim et al., 2007). This is likely because several hundred males and females are usually reared per generation (Chad Nielson, 2008, pers. commun.) in the laboratory, which may correspond to some hundreds of effective breeders. Only the non-diapause colony (over 190 generations in colony) showed a moderate (15-39\%) loss in genetic variability. There was little evidence that the laboratory colonies of $D$. $v$. virgifera had undergone significant genetic bottlenecks or selection processes (Kim et al., 2007), as one would expect from other insects (Rossler, 1975; Diamond et al., 2010).

\section{Differences between colonies from genetically different populations}

The origins of the genetic populations studied here (CSE European, NW Italian, Central-to-Northern USA) affected the average levels and variability of some phenotypic traits. Such effects of a population's origin are easier to explain than changes due to laboratory rearing. This is because CSE European and NW Italian populations are both expanding populations that originated from two independent introductions from the USA (Miller et al., 2005; Ciosi et al., 2008). During the separate introductions, multiple independent genetic bottlenecks occurred because of multiple independent founder effects (Facon et al., 2006). This resulted in the partition of the genetic variability of the source population (i.e., USA), among the invasive populations that are thus genetically differentiated (Ciosi et al., 2008), which is consequently also reflected in some of their phenotypic traits.

\section{Conclusions}

In summary, none of the 16 tested traits, with the possible exception of crawling behaviours, appeared to be consistently stable across tested colonies. All the traits were either influenced by the genetic characteristics of the original population, the original conditions in the collection field and/or the subsequent laboratory rearing. Our data suggest that activity can be studied with field-collected $D$. v. virgifera as well as with colonies reared in laboratory for a few generations, whereas the use of such insects is less advised for comparative research about fitness or morphometrics (table 6). Care should be taken when individuals from long-term reared laboratory colonies are used for behavioural studies. Average levels of morphometric and fitness traits, however, seem to be always prone to change due to rearing. However; the variability in those traits remains relatively stable if enough specimens are used in rearing cycles.

In conclusion, these results should only be used as guidelines. They 'only' provide estimations about the potential for changes in traits that may occur due to influences from field conditions or during laboratory-rearing. There is still much that can be done and learned by comparing populations at the organismal level. Nevertheless, we believe that these results can help researchers determine whether their planned investigations of $D$. $v$. virgifera fitness, activity, or morphometrics should be conducted with $D$. $v$. virgifera from laboratory colonies or with field-collected specimens.

\section{Acknowledgements}

We gratefully acknowledge Marc Ciosi and Xavier Fauvergue (INRA, Sophia Antipolis, France) for helpful comments during the planning period of experiments, and the Diabrotica team assisting Chad Nielson at USDA-ARS North Central Agricultural Research Laboratory, Brookings, 
South Dakota, USA for technical rearing advice and for providing USA colonies. We thank Benoit Facon (INRA, Centre de Biologie et de Gestion des Populations, Montferrier, France), Nick Mills (University of California, Berkeley, USA), Mark Szalai (University of Godollo, Hungary), Christian Lexer (University of Fribourg, Switzerland), and Gerald Bermond (INRA UMR, Sophia Antipolis, and University of Nice, France) for statistical advice. We thank Stefan Vidal (University of Goettingen, Germany) and an anonymous reviewer for their valuable comments and improvements of the manuscript. This research was funded by the French 'Agence Nationale de la Recherche' (ANR-06-BDIV-008).

\section{References}

Begley, C.G. (2013) Six red flags for suspect work. Nature 497, 433-434.

Benjamini, Y. \& Hochberg, Y. (1995) Controlling the false discovery rate: a practical and powerful approach to multiple testing. Journal of the Royal Statistical Society 57, 289-300.

Bernardo, J. (1996) Maternal effects in animal ecology. American Zoologist 36, 83-105.

Boiler, E.F. \& Chambers, D.L. (1977) Quality control of massreared insects. pp. 219-236 in Ridgway, R.I. \& Vinson, S.B. (Eds) Biological Control of Insects by Augmentation of Natural Enemies. New York, USA, Plenum Press. 480 pp.

Branson, T.F. (1976) The selection of a non-diapause strain of Diabrotica virgifera (Coleoptera: Chrysomelidae). Entomologia Experimentalis et Applicata 19, 148-154.

Branson, T.F. \& Jackson, J.J. (1988) An improved diet for adult Diabrotica virgifera virgifera (Coleoptera: Chrysomelidae). Journal Kansas Entomological Society 61, 353-355.

Branson, T.F. \& Johnson, R.D. (1973) Adult western corn rootworms: oviposition, fecundity, and longevity in the laboratory. Journal of Economic Entomology 66, 417-418.

Branson, T.F. \& Krysan, J.L. (1981) Feeding and oviposition behaviour and life cycle strategies of Diabrotica: an evolutionary view with implications for pest management. Environmental Entomology 10, 826-831.

Branson, T.F. \& Ortman, E.E. (1970) The host range of larvae of the western corn rootworm: further studies. Journal of Economic Entomology 63, 800-803.

Branson, T.F. \& Sutter, G.R. (1985) Influence of population density of immatures on size, longevity, and fecundity of adult Diabrotica virgifera virgifera (Coleoptera: Chrysomelidae). Environmental Entomology 14, 687-690.

Branson, T.F., Guss, P.L., Krysan, J.L. \& Sutter, G.R. (1975) Corn Rootworms: Laboratory Rearing and Manipulation. Peoria, IL, USA, ARS-NC-28, ARS USDA.

Bush, G.L., Neck, R.W. \& Kitto, G.B. (1976) Screwworm eradication, inadvertent selection for non-competitive ecotypes during mass rearing. Science 193, 491-493.

Chambers, D.L. (1977) Quality control in mass-rearing. Annual Review of Entomology 22, 2851-308.

Chiang, H.C. (1973) Bionomics of the northern and western corn rootworms. Annual Review of Entomology 18, 47-72.

Ciosi, M., Miller, N.J., Kim, K.S., Giordano, R., Estoup, A. \& Guillemaud, T. (2008) Invasion of Europe by the western corn rootworm, Diabrotica virgifera virgifera: multiple transatlantic introductions with various reductions of genetic diversity. Molecular Ecology 17, 3614-3627.

Coats, S.A., Tollefson, J.J. \& Mutchmor, J.A. (1986) Study of migratory flight in the western corn rootworm
(Diabrotica virgifera virgifera) (Coleoptera: Chrysomelidae). Environmental Entomology 15, 620-625.

Culy, M.D., Edwards, C.R. \& Cornelius, J.R. (1992) Effect of silk feeding by western corn rootworm (Coleoptera: Chrysomelidae) on yield and quality of inbred corn in seed corn production fields. Journal of Economic Entomology 85, 2440-2446.

Diamantidis, A.D., Carey, J.R., Nakas, Ch.T. \& Papadopoulos, N. T. (2011) Ancestral populations perform better in a novel environment: domestication of Mediterranean fruit fly populations from five global regions. Biological Journal of Linnaean Society 102, 334-345.

Diamond, S.E., Hawkins, S.D., Nijhout, H.F. \& Kingsolver, J.G. (2010) Evolutionary divergence of field and laboratory populations of Manduca sexta in response to host plant quality. Ecological Entomology 35, 166-174.

Duan, J.J., Weber, D.C. \& Dorn, S. (1998) Flight behaviour of preand postdiapause apple blossom weevils in relation to ambient temperature. Entomologia Experimentalis et Applicata 88, 97-99.

Facon, B., Genton, B.J., Shykoff, J., Jarne, P., Estoup, A. \& David, P. (2006) A general eco-evolutionary framework for understanding bioinvasions. Trends in Ecology and Evolution 21, 130-135.

Fox, C.W. \& Czesak, M.E. (2006) Selection of body size and sexual dimorphism differs between host species in a seed-feeding beetle. Journal of Evolutionary Biology 19, 1167-1174.

Games, P.A. \& Howell, J.F. (1976) Pairwise multiple comparison procedures with unequal $n$ 's and/or variances: a Monte Carlo study. Journal of Educational Statistics 1, 113-125.

George, B.W. \& Ortman, E.E. (1965) Rearing the western corn rootworm in the laboratory. Journal of Economic Entomology 58, 375-377.

Gillette, C.P. (1912) Diabrotica virgifera as a corn rootworm. Journal of Economic Entomology 5, 364-366.

Hammack, L. \& French, B.W. (2007) Sexual dimorphism of basitarsi in pest species of Diabrotica and Cerotoma (Coleoptera : Chrysomelidae). Annual Review of Entomological Society of America 100, 59-63.

Hill, R.E. (1975) Mating, oviposition patterns, fecundity and longevity of the western corn rootworm. Journal of Economic Entomology 68, 311-315.

Hill, W.G. \& Caballero, A. (1992) Artificial selection experiments. Annual Review Ecological Systems 23, 287-310.

Huettel, M.D. (1976) Monitoring the quality of laboratory reared insects: a biological and behavioural perspective. Environmental Entomology 5, 807-814.

Jenner, W.H. \& Kuhlmann, U. (2006) Significance of host size for a solitary endoparasitoid: a trade-off between fitness parameters. Basic and Applied Ecology 7, 461-471.

Johnston, J.A., Donovan, L.A. \& Arnold, M.L. (2004) Novel phenotypes among early generation hybrids of two Louisiana iris species: flooding experiments. Journal of Ecology 92, 967-976.

Khazaeli, A.A. \& Curtsinger, J.W. (2010) Life history variation in an artificially selected population of Drosophila melanogaster: Pleiotropy, superflies, and age-specific adaptation. Evolution 64, 3409-3416.

Kim, K.S. \& Sappington, T.W. (2005) Genetic structuring of western corn rootworm (Coleoptera: Chrysomelidae) populations in the United States based on microsatellite loci analysis. Environmental Entomology 34, 494-503.

Kim, K.S., French, B.W., Summerford, D. \& Sappington, T.W. (2007) Genetic diversity in laboratory colonies of western 
corn rootworm (Coleoptera: Chrysomelidae), including a non-diapause colony. Environmental Entomology 36, 637-645.

Kinnear, P.R. \& Gray, C.D. (2000) SPSS for Windows Made Simple. East Sussex, UK, Psychology Press Ltd.

Kiss, J., Edwards, C.R., Berger, H.K., Cate, P., Cean, M., Cheek, S., Derron, J., Festic, H., Furlan, L., Igrc-Barcic, J., Ivanova, I., Lammers, W., Omelyuta, V., Princzinger, G., Reynaud, P., Sivcev, I., Sivicek, P., Urek, G. \& Vahala, O. (2005) Monitoring of western corn rootworm (Diabrotica virgifera virgifera LeConte) in Europe 1992-2003. pp. 29-39 in Vidal, S., Kuhlmann, U. \& Edwards, C.R. (Eds) Western Corn Rootworm: Ecology and Management. Wallingford, UK, CABI Publishing.

Krebs, C.J. (1994) Ecology: the Experimental Analysis of Distribution and Abundance. New York, USA, Harper Collins.

Krysan, J.L. (1972) Embryonic stages of Diabrotica virgifera (Coleoptera: Chrysomelidae) at diapause. Annals of the Entomological Society of America 65, 768-769.

Krysan, J.L. (1982) Diapause in the Nearctic species of the virgifera group of Diabrotica: evidence for tropical origin and temperate adaptations. Annals of the Entomological Society of America 75, 136-142.

Krysan, J.L. \& Miller, T.A. (1986) Methods for Study of Pest Diabrotica. New York, Springer. p. 260.

Krysan, J.L. \& Smith, R.F. (1987) Systematics of the virgifera species group of Diabrotica (Coleoptera: Chrysomelidae: Galerucinae). Entomography 5, 375-484.

Kuhar, T.P. \& Youngman, R.R. (1995) Sex ratio and sexual dimorphism in western corn rootworm (Coleoptera: Chrysomelidae) adults on yellow sticky traps in corn. Environmental Entomology 24, 1408-1413.

Lande, R. \& Arnold, S.J. (1983) The measurement of selection on correlated characters. Evolution 37, 1210-1226.

Lefko, S.A., Nowatzki, T.M., Thompson, S.D., Binning, R.R., Pascual, M.A., Peters, M.L., Simnbro, E.J. \& Stanley, B.H. (2008) Characterizing laboratory colonies of western corn rootworm (Coleoptera: Chrysomelidae) selected for survival on maize containing event DAS-59122-7. Journal of Applied Entomology 132, 189-204.

Levine, E. \& Oloumi-Sadeghi, H. (1991) Management of Diabroticite Rootworms in corn. Annual Review of Entomology 36, 229-255.

Levine, E., Spencer, J.L., Isard, S.A., Onstad, D.W. \& Gray, M.E. (2002) Adaptation of the western corn rootworm to crop rotation: evolution of a new strain in response to a management practice. American Entomologist. 48, 94-107.

Li, H., Toepfer, S. \& Kuhlmann, U. (2009) Relationship between phenotypic traits and selected fitness components of Diabrotica virgifera virgifera. Entomologia Experimentalis et Applicata 131, 254-263.

Li, H., Toepfer, S. \& Kuhlmann, U. (2010) Flight and crawling activities of Diabrotica virgifera virgifera (Coleoptera: Chrysomelidae) in relation to morphometric traits. Journal of Applied Entomology 134, 449-461.

Mabry, T.R., Spencer, J.L., Levine, E. \& Isard, S.A. (2004) Western corn rootworm (Coleoptera: Chrysomelidae) behaviour is affected by alternating diets of maize and soybean. Environmental Entomology 33, 860-871.

Masson, L.J., Pashley, D.P. \& Johnson, S.J. (1987) The laboratory as an altered habitat: phenotypic and genetic consequences of colonization. Florida Entomologist 70, 49-58.

Meihls, L.N., Higdon, M.L., Siegfried, B., Miller, N.J., Sappington, T.W., Ellersieck, M.R., Spencer, T.A.
\& Hibbard, B.E. (2008) Increased survival of western corn rootworm on transgenic corn within three generations of on-plant greenhouse selection. Proceedings of the National Academy of Sciences of the United States of America 105, 19177-19182.

Miller, N.J., Estoup, A., Toepfer, S., Bourguet, D., Lapchin, L., Derridj, S., Kim, K.S., Reynaud, P., Furlan, L. \& Guillemaud, T. (2005) Multiple transatlantic introductions of the western corn rootworm. Science 310, 992.

Miller, N.J., Kim, K.S., Ratcliffe, S.T., Estoup, A., Bourguet, D. \& Guillemaud, T. (2006) Absence of genetic divergence between western corn rootworms (Coleoptera: Chrysomelidae) resistant and susceptible to control by crop rotation. Journal of Economic Entomology 99, 685-690.

Miller, N.J., Ciosi, M., Sappington, T.W., Ratcliffe, S., Spencer, J.L. \& Guillemaud, T. (2007) Genome scan of Diabrotica virgifera virgifera for genetic variation associated with crop rotation tolerance. Journal Applied Entomology 131, 378-385.

Miller, N.J., Guillemaud, T., Giordano, R., Siegfried, B.D., Gray, M.E., Meinke, L.J. \& Sappington, T.W. (2009) Genes, gene flow and adaptation of Diabrotica virgifera virgifera. Agricultural and Forest Entomology 11, 47-60.

Miyatake, T. \& Yamagishi, M. (1999) Rapid evolution of larval development time during mass-rearing in the melon fly, Bactrocera cucurbitae. Research Population Ecology 41, 291-297.

Mohaghegh, J., de Clercq, P., Tirry, L. (1999) Effects of rearing history and geographical origin on reproduction and body size of the predator Podisus nigrispinus (Heteroptera: Pentatomidae). European Journal of Entomology 96, 69-72.

Modic, S., Schoers, H.J. \& Urek, G. (2008) Survival of eggs of D. v. virgifera during Winter and Isolation of their Fungal Enemies. in Conference on Western Corn Rootworm: Research and Long Term Management Needs for the European Maize Production, Symposium on western corn rootworm ecology and management, 25-29 May 2008, Gottingen, Germany.

Moeser, J. \& Hibbard, B.E. (2005) A synopsis of the nutritional ecology of larvae and adults of Diabrotica virgifera virgifera in the new and old world - nouvelle cuisine for the invasive maize pest Diabrotica virgifera virgifera in Europe? pp. 41-65 in Vidal, S., Kuhlmann, U. \& Edwards, C.R. (Eds) Western Corn Rootworm: Ecology and Management. Wallingford, UK, CABI Publishing.

Mousseau, T.A. \& Fox, C.W. (1998) Maternal Effects as Adaptations. New York, Oxford University Press.

Norris, D.E., Shurtleff, A.C., Toure, Y.T. \& Lanzaro, C. (2001) Microsatellite DNA polymorphism and heterozygosity mong field and laboratory populations of Anopheles gambiaes. s. (Diptera: Culicidae). Journal of Medical Entomology 38, 336-340.

Oswald, K., French, B.W., Nielson, C. \& Bagley, M. (2011) Selection for Cry3Bb1 resistance in a genetically diverse population of non-diapausing western corn rootworm (Coleoptera: Chrysomelidae). Journal of Economic Entomology 104, 1038-1044.

Prokopy, R.J., Economopoulos, A.P. \& McFadden, M.W. (1975) Attraction of wild and laboratory-cultured Dacus oleae flies to small rectangles of different hues, shades, and tints. Entomologia Experimentalis et Applicata 18, 141-152.

R Development Core Team (2009) R: A Language and Environment for Statistical Computing. R Foundation for Statistical Computing, Vienna, Austria. Available online at http://www.R-project.org (accessed May 2009). 
Richerson, J.V. \& Cameron, E.A. (1974) Differences in pheromone release and sexual behaviour between laboratory-reared and wild gypsy moth adults. Environmental Entomology 3, 475-481.

Rossler, Y. (1975) The ability to inseminate: a comparison between laboratory-reared and field populations of the Mediterranean fruit fly (Ceratitis capitata). Entomologia Experimentalis et Applicata 18, 255-260.

Scannapieco, A.C., Sambucetti, P. \& Norry, F.M. (2009) Direct and correlated responses to selection for longevity in Drosophila buzzatii. Biological Journal Linnaean Society 97, 738-748.

Singh, P. \& Moore, R.F. (1999) Handbook of Insect Rearing. Amsterdam, Netherlands, Elsevier.

Sivcev, I., Manojlovic, B., Krnjajic, S., Dimic, N., Draganic, M., Baca, F., Kaitovic, Z., Sekulic, R. \& Keresi, T. (1994) Distribution and harmfulness of Diabrotica virgifera LeConte (Coleoptera, Chrysomelidae), a new maize pest in Yugoslavia. Zashita Bilja 45, 19-26.

Spencer, J.L., Hibbard, B.E., Moeser, J. \& Onstad, D.W. (2009) Behaviour and ecology of the western corn rootworm (Diabrotica virgifera virgifera LeConte). Agricultural and Forest Entomology 11, 9-27.

Spurgeon, D.W. (2012) Physiological consequences of laboratory rearing of Lygus hesperus (Hemiptera: Miridae). Environmental Entomology 41, 415-419.

Staetz, C.A., Ball, H.J. \& Carlson, S.D. (1976) Antennal morphology of Diabrotica virgifera adults (Coleoptera: Chrysomelidae). Annual Review of the Entomological Society of America 69, 695-698.

Stearns, S.C. (1992) The Evolution of Life Histories. Oxford, UK, Oxford University Press.
Storey, J.D. (2002) A direct approach to false discovery rates. Journal of the Royal Statistical Society 64, 479-498.

Storey, J.D. \& Tibshirani, R. (2003) Statistical significance for genome-wide experiments. Proceeding of the National Academy of Sciences USA 100, 9440-9445.

Tingey, W.M. (1986) Techniques for evaluating plant resistance to insects. pp. 251-284 in Miller, J.R. \& Miller, T.A. (Eds) Insectplant Interactions. New York, USA, Springer.

Toepfer, S. \& Kuhlmann, U. (2006) Constructing life-tables for the invasive maize pest Diabrotica virgifera virgifera (Col.; Chrysomelidae) in Europe. Journal of Applied Entomology 130, 193-205.

Toepfer, S., Levay, N. \& Kiss, J. (2005) Suitability of different fluorescent powders for mass-marking the Chrysomelid, Diabrotica virgifera virgifera LeConte. Journal of Applied Entomology 129, 456-464.

Toepfer, S., Li, H., Guillemaud, Th., Wang, Zh. \& Wu, K. (2012) Bioassay arenas for studying the biology and ecology of adult western corn rootworm. IOBC/IWGO Newsletter.

Valladares, F., Sanchez-Gomez, D. \& Zavala, M.A. (2006) Quantitative estimation of phenotypic plasticity: bridging the gap between the evolutionary concept and its ecological applications. Journal of Ecology 94, 1103-1116.

VanWoerkom, G.J., Turpin, F.T. \& Barrett, J.R. Jr. (1980) Influence of constant and changing temperatures on locomotor activity of adult western corn rootworms (Diabrotica virgifera) in the laboratory. Environmental Entomology 9, 32-34.

Vidal, S., Kuhlmann, U. \& Edwards, C.R. (2005) Western Corn Rootworm: Ecology and Management. Wallingford, UK, CABI Publishing, p. 310. 